\title{
A proficiency test system to improve performance of milk analysis methods and produce reference values for component calibration samples for infrared milk analysis ${ }^{1}$
}

\author{
Karen L. Wojciechowski, Caterina Melilli, and David M. Barbano ${ }^{2}$ \\ Department of Food Science, Cornell University, Northeast Dairy Foods Research Center, Ithaca, NY 14853
}

\begin{abstract}
Our goal was to determine the feasibility of combining proficiency testing, analytical method qualityassurance system, and production of reference samples for calibration of infrared milk analyzers to achieve a more efficient use of resources and reduce costs while maximizing analytical accuracy within and among milk payment-testing laboratories. To achieve this, we developed and demonstrated a multilaboratory combined proficiency testing and analytical method quality-assurance system as an approach to evaluate and improve the analytical performance of methods. A set of modified milks was developed and optimized to serve multiple purposes (i.e., proficiency testing, quality-assurance and method improvement, and to provide reference materials for calibration of secondary testing methods). Over a period of years, the approach has enabled the group of laboratories to document improved analytical performance (i.e., reduced within- and between-laboratory variation) of chemical reference methods used as the primary reference for calibration of high-speed electronic milk-testing equipment. An annual meeting of the laboratory technicians allows for review of results and discussion of each method and provides a forum for communication of experience and techniques that are of value to new analysts in the group. The monthly proficiency testing sample exchanges have the added benefit of producing all-laboratory mean reference values for a set of 14 milks that can be used for calibration, evaluation, and troubleshooting of calibration adjustment issues on infrared milk analyzers.
\end{abstract}

Received January 24, 2016.

Accepted April 2, 2016.

${ }^{1}$ Use of names, names of ingredients, and identification of specific models of equipment is for scientific clarity and does not constitute any endorsement of product by authors by Cornell University or the Northeast Dairy Foods Research Center.

${ }^{2}$ Corresponding author: dmb37@cornell.edu
Key words: ether extraction, Kjeldahl, proficiency testing

\section{INTRODUCTION}

Laboratory performance proficiency testing (Thompson and Wood, 1993) of analytical method performance has become an integral part of third-party performance certification programs for laboratories around the world. A group of metrics [all-laboratory mean, within-laboratory repeatability standard deviation $\left(\mathbf{S}_{\mathrm{r}}\right)$, between-laboratory reproducibility standard deviation $\left(\mathbf{S}_{\mathbf{R}}\right)$, repeatability limit $\left(\mathbf{r}\right.$-value $\left.=2.8 \times \mathrm{S}_{\mathrm{r}}\right)$, reproducibility limit $\left(\mathbf{R}\right.$-value $\left.=2.8 \times \mathrm{S}_{\mathrm{R}}\right)$, Z-scores, laboratory ranking, Euclidean distance plots, Pareto diagrams, and so on] and data analysis tools [statistical outlier removal procedures (Horwitz, 1995; Lynch, 1998) to create an all-laboratory mean with outliers removed] can be used to evaluate the results of proficiency testing of a population of laboratories. Proficiency testing results indicate if the analytical performance of a laboratory on a particular method and analyte are within some normative performance criteria (such as method performance in an interlaboratory study for method validation) and provides a comparison to the performance of other laboratories. However, to achieve excellent performance of analytical methods, each individual laboratory needs to have a quality-assurance system in place for in-house control of each method performance and to detect gradual changes in house analytical procedures (sometimes called method creep) used to carry each method. When a large group of laboratories have a common need in quality-assurance and proficiency testing on methods performed in all laboratories, then an opportunity exists for integration of proficiency testing and multilaboratory qualityassurance that can be used to improve the analytical performance and control of all laboratories within the group. Taking the previous idea one step further, the approach could be used to produce reference values 
on sample materials using in a proficiency test sample exchange, particularly biological materials that have a relatively short shelf-life. An opportunity for such a system exists in milk payment testing laboratories, where the results of analysis of large numbers of samples are used to establish value of large amounts of milk and milk products that are bought and sold on a daily basis. The analytical accuracy of those results can have a large effect on both buyers and sellers of dairy products (Lynch et al., 2004). Our objectives were to use data from monthly chemical analyses of a set of 14 milks with an orthogonal matrix of composition to monitor the analytical performance of the reference chemical analysis methods, to evaluate and improve the analytical proficiency of individual laboratories conducting these reference methods, and to calculate all-laboratory mean reference values for fat, protein, lactose, and TS for these milks that would enable the proficiency test samples to be used as reference materials to calibrate a high-speed infrared milk analysis method for measurement of milk composition.

\section{MATERIALS AND METHODS}

\section{Rationale and Approach}

Milk payment-testing laboratories have a need for high-quality reference standards to calibrate infrared milk analyzers (Kaylegian et al., 2006a). Many paymenttesting laboratories also analyze milks using classical chemical methods. Milk payment laboratories have a need for quality-assurance control of both chemical reference methods and secondary instrumental methods to ensure accuracy of testing. Often, calibration samples and samples used for proficiency and quality-assurance programs for testing methods are separate samples and separate analyses. Our goal was to determine the feasibility of combining these 2 activities to achieve a more efficient use of resources and reduce costs while maximizing analytical performance within and between laboratories. Prior to 2004, the samples used to evaluate chemical method performance were individual farm milks. It is sometimes difficult to achieve a good range of concentrations of each milk component and a good distribution of concentration levels within the range when using individual farm milks. Starting in 2004, a program was initiated to formulate an improved set of milks for calibration of infrared milk analyzers, and at the same time improve the performance of reference testing methods. A set of 14 milks were formulated monthly to produce an orthogonal matrix of fat, protein, and lactose concentrations and were analyzed by a group of laboratories. Statistical outliers were removed and all-laboratory mean reference values and within- and between-laboratory variation (i.e., $\mathrm{s}_{\mathrm{r}}$ and $\mathrm{s}_{\mathrm{R}}$ ) with outliers removed were calculated for each milk for each component (fat, protein, lactose, and TS) per laboratory. The proficiency of the labs conducting the chemical reference methods was evaluated utilizing Z-scores (Thompson and Wood, 1993), Pareto diagrams (Hubbard, 1990), and Euclidian distance plots (Kaylegian et al., 2006b) without outliers removed. We analyzed residual plots of differences between the all-laboratory mean reference values with outliers removed for each sample and the actual values without outliers removed plotted as a function of component concentration for each laboratory and method (i.e., ether extraction, Kjeldahl true protein, spectrophotometric enzymatic lactose, forced-air atmospheric oven-drying for TS). This approach allowed us to characterize analytical deviations from the mean of all laboratories for each laboratory for each method, to determine the causes of deviations, and to recommend possible corrections and improvements that could be made.

\section{Modified Milks}

Manufacture and Design of Sample Set. The manufacture of the 14 modified milk samples was done in the Cornell University pilot plant described by Kaylegian et al. (2006a). These modified milks were designed for use in calibration of infrared milk analyzers (Kaylegian et al., 2006b). Pasteurized whole milk $\left(72^{\circ} \mathrm{C}\right.$ for $15 \mathrm{~s}$ ) was gravity separated overnight at $4^{\circ} \mathrm{C}$. Control of temperature and time is very important because too much heat will prevent gravity separation from occurring (Geer and Barbano, 2014a,b). The gravity skim layer ( $90 \%$ by weight) was drained from the bottom of the tank first, followed by removal of the cream in 5 or 6 layers. Each of the cream layers was analyzed for fat content, and selected layers were blended to create a cream ingredient with a fat content of 22 to $27 \%$. A centrifugal cream separator was used to further reduce the fat content of the gravity skim to $<0.07 \%$. The centrifugally separated skim milk was ultrafiltered to obtain UF retentate (concentration factor $2 \times$ ) and permeate. The cream ingredient, skim UF permeate, skim UF retentate, reagent-grade $\alpha$-lactose monohydrate (MultiPharm, EM Science, Gibbstown, NJ), and laboratory-grade water were blended to create 14 calibration samples with a broad range and an orthogonal matrix of component concentrations (Kaylegian et al., 2006a). Samples were preserved (Barbano et al., 2010) with Microtabs II (Advanced Instruments, Norwood, $\mathrm{MA})$, split into vials, and refrigerated $\left(4^{\circ} \mathrm{C}\right)$. The modified milk calibration sets used were produced at Cornell University and shipped with wet ice overnight to each of the participating laboratories for analysis. Samples 
were analyzed using reference chemistry methods by a group of laboratories for fat, true protein, TS, and lactose.

Chemical Analysis. Chemical analyses of all calibration samples were conducted using the following AOAC International (2000) methods: fat by modified Mojonnier ether extraction (method 989.05), true protein by Kjeldahl analysis (method 991.22), TS by oven-drying (method 990.20), and anhydrous lactose determined by enzyme analysis (method 2006.06) modified to measure lactose by weight instead of volume and corrected for cuvette path length with the results expressed as anhydrous lactose (Lynch et al., 2007). This network of laboratories, for the most part, was the same that conducted the collaborative studies for each of the methods for the milk matrix: Mojonnier ether extraction (Barbano et al., 1988), Kjeldahl true protein (Barbano et al., 1990, 1991), anhydrous lactose by an spectrophotometric enzyme assay (Lynch et al., 2007), and forced-air, oven-drying TS (Clark et al., 1989).

\section{Statistical Methods}

Outlier Data Removal Procedures. In the context of this work, one of our primary goals was to produce reference values for each sample that reflect as closely as possible the true value for the concentration of each component in a sample. This is a different goal than the goal of collaborative study of a method. In a collaborative study, characterization of the within- and betweenlaboratory method performance statistics is the goal and the mean values for each sample are of little interest. Thus, the perspective in the removal of outliers is a little different when the goal is to produce reference values for each sample that are the best estimate of the true value for the concentration of each component in each sample. This is particularly true when a set of reference values will be used to calibrate many high-speed electronic milk-testing instruments that will influence the payment for very large volumes of milk.

The basis for the decision to remove an outlier, particularly in the Cochran test, is not as well defined as it could be. In the instructions to collaborative Study Directors (AOAC International, 2000) for outlier removal from a data set in a collaborative study, the following general guidance is provided: "The decision as to whether a value(s) should be removed as an outlier ultimately is not statistical in nature. The decision must be made by the Study Director on the basis of the indicated probability given by the outlier test and any other information that is pertinent. (However, for consistency with other organizations adhering to the harmonized outlier removal procedure, the estimate resulting from rigid adherence to the prescribed pro- cedure should be reported.)" (Appendix D of AOAC International 2000). Thus, the importance becomes very clear: to remove or not to remove is the question.

The harmonized outlier removal procedure is a sequential statistical process utilizing the Cochran, single Grubbs, or double Grubbs procedures, in that order (AOAC International 2000). This outlier removal procedure uses an iterative process to establish the outlier data that should be removed. Data from a minimum of 8 laboratories is required for each sample material to avoid unduly large confidence bands about the estimated parameters. In our study the Cochran outlier test was used for removal of laboratories (or indirectly for removal of single extreme individual values) from a set of laboratory values that show significantly greater variability among replicate (within-laboratory) analyses than the other laboratories and applied as a 1-tail test at a probability of $2.5 \%$. Table 1 contains 5 examples of outlier removal decisions and illustrates the importance of decisions made for removal of Cochran outliers. In Table 1, the data for sample 1 illustrates that laboratory 5 was identified as a Cochran outlier, but should we remove the data for both replicates or only one replicate for laboratory 5 ? Replicate 2 of laboratory 5 was outside the range of all the other values for sample 1 and was clearly an outlier, but replicate 1 for laboratory 5 is within the range of all the other values for all labs. Removal of both values for laboratory 5 would reduce the number of labs to 8 , whereas removal of only replicate 2 would leave the number of laboratories at 9 going into the single Grubbs test. The Grubbs test detects outlier laboratories and we noted an advantage of having more laboratories (9 vs. 8) for the Grubbs test.

Laboratory 2, sample 2 was identified as a Cochran outlier (Table 1). Upon examination of the 2 values for laboratory 2, it was determined that the replicate 1 was lower than all other values and replicate 2 was higher than all other values; therefore, both values were removed as Cochran outliers. Eight labs remained and a single Grubbs test was performed and no Grubbs outliers were detected. The Grubbs tests for removal of laboratories with extreme averages were applied in the following order: single Grubbs test (2-tail; $P=2.5 \%$ ) and then, if no outlier was found, double Grubbs test (2 values at the highest end, 2 values at the lowest end, and 2 values, 1 at each end, at an overall $P=$ $2.5 \%$ ) was applied (Appendix D in AOAC International 2000). If the single Grubbs test signals the need for removal of an outlier laboratory, then the single Grubbs outlier laboratory was removed and the Cochran test was rerun.

In Table 1, sample 3, laboratories 4 and 6 were identified as Cochran outlier laboratories. Replicate 1 for 
laboratory 4 was the highest of all values for sample 3 and replicate 1 for laboratory 6 was the lowest value for all laboratories. The replicate 2 values for laboratories 4 and 6 are both within the high-low range of all the other values for sample 3 . Therefore, only replicate 1 for both laboratories 4 and 6 was removed. This left data for 9 laboratories and it was possible to run the Grubbs tests. If both values from laboratories 4 and 6 had been removed, then only been 7 laboratories would remain and it would not have been appropriate (i.e., $<8$ laboratories) to run the Grubbs test. The single Grubbs test was performed on all 9 laboratories and no further outliers were detected for sample 3.

Cochran outliers were removed from sample 4 (replicates 1 , labs 4 and 6 ) the same as for sample 3, except that, for the data in this example, the single Grubbs test detects laboratory 2 as a single high-outlier laboratory. Both replicates for laboratory 2 were removed, reducing the number of laboratories to 8 , allowing the single Grubbs test to be run again. Another single Grubbs high-outlier laboratory was identified (laboratory 8) and removed. At this point only 7 laboratories remained for sample 4 and no further outlier tests could be done for sample 4 . However, when laboratory 4 and 6 (replicates 1 and 2) outlier values were removed, the all-laboratory mean was 3.5358. Choosing not to remove the replicate 2 values for laboratory 4 and 6 allowed the single Grubbs test to be run and removed labs 2 and 8 as outliers. This made a large difference in the all-laboratory mean reference value for sample 4 (i.e., if replicate 1 and 2 of laboratory 4 and 6 and no Grubbs outliers were removed because of too few laboratories, then all-laboratory mean $=3.5322$; if only replicate 1 of 4 and 6 , then labs 2 and 8 were removed as single Grubbs high-outliers, then all-laboratory mean $=3.5041)$.
In the data for sample 5 in Table 1 , the removal of Cochran outliers was the same as in samples 3 and 4 , maintaining 9 laboratories going into the single Grubbs test. Nine labs were evaluated by single Grubbs test, however, and no single Grubbs outliers were detected. A double Grubbs test must be performed on the data for the 9 remaining laboratories to determine if the single Grubbs test was masked because there were either 2 high, 2 low, or single high- and low-outlier laboratories. Laboratories 2 and 8 were identified as double Grubbs high outliers. With the removal of replicates 1 and 2 for laboratory 2 and 8 , and removal of only the replicate 1 Cochran outliers, the all-laboratory mean became 3.5089. If the Cochran outlier laboratories 4 and 6 were removed completely (i.e., both replicate 1 and 2 ), the mean value would have been 3.5555 for sample 5 . This made a large difference in the all-laboratory mean reference value for sample 5 and illustrates the importance of choosing not to remove the replicate 2 values for laboratories 4 and 6 as Cochran outliers so that the Grubbs tests could be run. A double Grubbs outlier pair of laboratories is present if the selected value for the percentage decrease from the original exceeds the critical value listed in the double Grubbs value table at the $P=2.5 \%$ level. If this second test is positive, the 2 values responsible for activating the test must be removed and the Cochran test rerun, then repeat the sequence of Cochran, single Grubbs, double Grubbs. This was the case for sample 5, as laboratories 2 and 8 were both high-outlier laboratories and they were identified as double Grubbs high outliers. This reduces the number of laboratories to 7 and no further outlier test should be done. Outlier removal should stop before more than 2 out of 9 laboratories are removed. If no outliers are removed for a given cycle, the outlier removal is complete.

Table 1. Examples of test results from individual laboratories that demonstrate outlier removal procedures Cochran, single Grubbs, and double Grubbs outliers and the decision-making required when establishing an all-laboratory mean reference value for each individual milk sample versus the use of outlier removal for the purpose of development of method performance statistics in a collaborative study of a method

\begin{tabular}{|c|c|c|c|c|c|c|c|c|c|c|}
\hline \multirow[b]{2}{*}{ Item } & \multicolumn{2}{|c|}{ Sample 1} & \multicolumn{2}{|c|}{ Sample 2} & \multicolumn{2}{|c|}{ Sample 3} & \multicolumn{2}{|c|}{ Sample 4} & \multicolumn{2}{|c|}{ Sample 5} \\
\hline & Replicate 1 & Replicate 2 & Replicate 1 & 1 Replicate 2 & Replicate 1 & Replicate 2 & Replicate 1 & Replicate 2 & Replicate 1 & Replicate 2 \\
\hline Lab 2 & 4.1003 & 4.0970 & $4.5314^{1}$ & $4.6412^{1}$ & 3.4997 & 3.5017 & $3.6701^{2}$ & $3.6704^{2}$ & $3.6701^{3}$ & $3.6704^{3}$ \\
\hline Lab 3 & 4.1054 & 4.1014 & 4.6025 & 4.6021 & 3.5178 & 3.5114 & 3.5112 & 3.5188 & 3.5112 & 3.5188 \\
\hline Lab 4 & 4.0977 & 4.0939 & 4.6078 & 4.6138 & $3.6922^{1}$ & 3.5014 & $3.6922^{1}$ & 3.5014 & $3.6922^{1}$ & 3.5014 \\
\hline Lab 5 & 4.0905 & $4.0619^{1}$ & 4.6045 & 4.6000 & 3.5002 & 3.4917 & 3.4921 & 3.5011 & 3.4921 & 3.5011 \\
\hline Lab 8 & 4.0876 & 4.0904 & 4.6027 & 4.6091 & 3.5107 & 3.5134 & $3.5601^{2}$ & $3.5651^{2}$ & $3.6721^{3}$ & $3.6714^{3}$ \\
\hline Lab 9 & 4.0973 & 4.1031 & 4.6312 & 4.6333 & 3.5020 & 3.5097 & 3.5114 & 3.5089 & 3.5462 & 3.5317 \\
\hline
\end{tabular}

${ }^{1}$ Cochran outlier.

${ }^{2}$ Single Grubbs high-outlier laboratory, first laboratory 2 and then laboratory 8 .

${ }^{3}$ Double Grubbs high-outlier laboratories. 
Within- and Between-Laboratory Method Performance. The statistical metric for within-laboratory variation of a method is the repeatability standard deviation $\left(\mathrm{S}_{\mathrm{r}}\right)$ and the metric for between-laboratory variation is the reproducibility standard deviation $\left(\mathrm{S}_{\mathrm{R}}\right)$. These values are commonly calculated as part of the process of method performance validation in collaborative studies (Horwitz, 1995). The calculation and practical use of these metrics in the laboratory was described by Lynch (1998). Our data were analyzed by the statistical procedures of AOAC International (2000) to determine the $\mathrm{S}_{\mathrm{r}}$, relative standard deviation of repeatability $\left(\mathbf{R S D}_{\mathbf{r}}\right)$, r-value, $\mathrm{S}_{\mathrm{R}}$, relative standard deviation of reproducibility $\left(\mathbf{R S D}_{\mathbf{R}}\right)$, and $\mathrm{R}$-value for each method, using the Microsoft Excel version of the AOAC International (Rockville, MD) Interlaboratory Statistical Program, version 1.11. These indices of method performance were produced previously in collaborative studies of the ether extraction method for fat determination (Barbano et al., 1988), the Kjeldahl method for CP determination (Barbano et al., 1990), and for true protein (Barbano et al., 1991), the forcedoven drying method for TS determination (Clark et al., 1989), and the enzymatic method for anhydrous lactose determination (Lynch et al., 2007). Proficiency-testing data, including method performance statistics, have been collected for several years and were compared with the method performance in the collaborative studies. A quality-assurance program for a network of laboratories within the USDA Federal Milk Markets was established with the goal of maintaining and improving the performance of this group of laboratories for these methods using various trouble-shooting approaches.

Laboratory Proficiency Evaluation. Pareto diagrams are bar graphs in which values are plotted in decreasing order of relative frequency. The diagrams are extremely useful for rapidly determining problems that need attention first. The purpose of the Pareto is to distinguish the "vital few from the trivial many" (Hubbard, 1990). Pareto diagrams of mean difference (MD), standard deviation of the difference (SDD), and Euclidean distance (ED) were used to identify laboratories that were having problems with a particular method, for ranking the performance of laboratories over time for each method, and for documenting performance improvement across time.

Euclidian distance plots are statistical measures of similarity that are the distance from an individual data point to the center point of a cluster of similar data (Massart et al., 1988), which is calculated using the MD and SDD from the all-laboratory mean for each sample. The ED was calculated as $E D=\sqrt{(M D)^{2}+(S D D)^{2}}$.
In this study, ED plots were used as a troubleshooting tool to identify laboratories that had high systematic or high random error using $t$-test values that indicated when the systematic error (i.e., bias) was too high for the amount of apparent random error displayed by that laboratory. The strategy that needs to be taken to help a laboratory improve performance is different depending on whether systematic or random error predominates in their proficiency test data.

The Z-score is a dimensionless quantity derived by subtracting the population mean (with statistical outliers removed) from an individual laboratory's (mean with no outliers removed) and then dividing the difference by the population long-term standard deviation of reproducibility. The long-term standard deviation of reproducibility is the $S_{R}$ from the original collaborative study for each method. Each reference method has a unique $S_{R}$ value for use in the Z-score calculations. Because Z-scores are dimensionless, they are useful to compare among all analytes, test materials, and analytical methods. Values obtained from diverse materials and concentration ranges can be, with caution, combined to give a composite score for a laboratory in one round of a proficiency test (Thompson and Wood 1993). Classifying the Z-scores to determine if a system is well-behaved or not is indicated by the following guidelines: the absolute value of $\mathrm{Z} \leq 2$ is satisfactory, the absolute value of $Z>2$ but $<3$ is questionable, and the absolute $\mathrm{Z}$-value $\geq 3$ is unsatisfactory (Thompson and Wood 1993). If the value for long-term reproducibility used in the Z-score calculation is a true representation of the current method performance, then $5 \%$ of the time one would expect Z-scores to exceed 3.

Troubleshooting Problems in Individual Laboratories. Through the use of Pareto diagrams of MD, SDD, and ED on a monthly basis, individual laboratories within each method that need the most help to improve performance can be identified. Examination of the ED plots for that laboratory help determine if the performance of the laboratory indicates high random error or high systematic bias. On a sample-by-sample basis within each laboratory, the all-laboratory mean for each sample (with outliers removed) is subtracted from that laboratory's mean value for each sample with no outliers removed. This produces a series of 14 differences from the all-laboratory mean for each method for each laboratory. These residual differences are plotted as a function of concentration for each analyte for each laboratory. Because of the 14 samples in each set, the set has a wide range of component concentration and uniform distribution of samples across the range of concentration; thus, it is possible to easily identify bias and slope behavior in the residual plots for a labora- 
tory. Depending on the method (i.e., ether extraction, Kjeldahl, enzymatic lactose, or oven-drying TS), observation of the behavior of the residual plot (i.e., systematic bias, systematic slope of residuals with change in concentration of component, or a combination of both) often leads to very specific identification of the points in the method that could be the cause of the deviations from the all-laboratory mean in that laboratory for that method. This information is communicated to the laboratory and method troubleshooting is done to achieve improved performance. The goal of the approach is to achieve improve performance of the laboratories (i.e., reduced $S_{r}$ and $S_{R}$ ) on these important reference methods that are used to establish reference values for samples that can be used to calibrate infrared milk analyzers used for payment testing. Examples of typical behavior of data and potential sources of the behavior will be presented.

\section{RESULTS AND DISCUSSION}

\section{Within- and Between-Laboratory Variation}

Ether Extraction. The indices of within- $\left(\mathrm{S}_{\mathrm{r}}\right)$ and between-laboratory $\left(\mathrm{S}_{\mathrm{R}}\right)$ variation that were established in the collaborative study (Barbano et al., 1988) of the modified Mojonnier ether extraction method are also shown in Table 2. The performance of the ether extraction method for fat content of milk was monitored over the period from 1989 to 1992 (Lynch et al., 1994) and 1995, 1996, 1998, and 1999 (Lynch et al., 2003) to determine if method performance was changing. Over the period from 1989 to 1999, 8 individual farm milks were tested in duplicate during each sample exchange. Each year, 1 or more representatives from each laboratory participating in these studies attended an annual workshop to review performance and focus on details

Table 2. Method performance statistics ${ }^{1}$ for repeatability and reproducibility of measurement of fat content of milk by modified Mojonnier ether extraction for the collaborative study 1988 and for selected years calculated from proficiency testing data

\begin{tabular}{|c|c|c|c|c|c|c|c|c|c|c|}
\hline Date & $\begin{array}{l}\text { Number of } \\
\text { laboratories }\end{array}$ & $\begin{array}{l}\text { Number } \\
\text { of tests }\end{array}$ & Mean & $\mathrm{S}_{\mathrm{r}}$ & $\mathrm{S}_{\mathrm{R}}$ & $\mathrm{RSD}_{\mathrm{r}}$ & $\mathrm{RSD}_{\mathrm{R}}$ & $\mathrm{r}$ & $\mathrm{R}$ & $\begin{array}{c}\text { Total } \\
\text { number of } \\
\text { outliers }\end{array}$ \\
\hline $1988^{2}$ & 10 & 2,520 & 3.886 & 0.0150 & 0.0200 & 0.3960 & 0.5120 & 0.0440 & 0.0560 & 178 \\
\hline $1989^{3}$ & 12 & 1,008 & 3.784 & 0.0090 & 0.0140 & 0.2410 & 0.3739 & 0.0260 & 0.0400 & 40 \\
\hline $1990^{3}$ & 14 & 1,162 & 3.639 & 0.0080 & 0.0130 & 0.2180 & 0.3560 & 0.0230 & 0.0370 & 33 \\
\hline $1991^{3}$ & 12 & 1,148 & 3.732 & 0.0070 & 0.0130 & 0.1890 & 0.3400 & 0.0200 & 0.0360 & 22 \\
\hline $1992^{3}$ & 14 & 1,148 & 3.686 & 0.0070 & 0.0130 & 0.1980 & 0.3390 & 0.0210 & 0.0350 & 34 \\
\hline $1995^{4}$ & 14 & 1,176 & 3.721 & 0.0068 & 0.0128 & 0.1835 & 0.3447 & 0.0191 & 0.0359 & 30 \\
\hline $1996^{4}$ & 14 & 1,190 & 3.810 & 0.0070 & 0.0122 & 0.1830 & 0.3188 & 0.0195 & 0.0341 & 42 \\
\hline $1998^{4}$ & 14 & 1,162 & 3.739 & 0.0072 & 0.0133 & 0.1922 & 0.3828 & 0.0201 & 0.0372 & 50 \\
\hline $1999^{4}$ & 14 & 1,204 & 3.733 & 0.0102 & 0.0137 & 0.2723 & 0.3675 & 0.0285 & 0.0384 & 60 \\
\hline 2007 & 11 & 3,528 & 3.001 & 0.0040 & 0.0085 & 0.1349 & 0.2833 & 0.0113 & 0.0238 & 94 \\
\hline 2008 & 10 & 3,472 & 3.032 & 0.0037 & 0.0080 & 0.1235 & 0.2648 & 0.0105 & 0.0225 & 86 \\
\hline 2009 & 9 & 3,024 & 3.037 & 0.0038 & 0.0078 & 0.1264 & 0.2577 & 0.0107 & 0.0219 & 101 \\
\hline 2010 & 9 & 3,059 & 3.028 & 0.0039 & 0.0073 & 0.1290 & 0.2412 & 0.0109 & 0.0204 & 102 \\
\hline 2011 & 9 & 2,940 & 2.979 & 0.0039 & 0.0073 & 0.1297 & 0.2452 & 0.0108 & 0.0204 & 79 \\
\hline 2012 & 10 & 3,472 & 2.970 & 0.0037 & 0.0078 & 0.1261 & 0.2642 & 0.0105 & 0.0220 & 117 \\
\hline 2013 & 10 & 3,472 & 2.959 & 0.0038 & 0.0078 & 0.1291 & 0.2630 & 0.0107 & 0.0218 & 119 \\
\hline Jan. 6, 2014 & 10 & 280 & 2.9542 & 0.0035 & 0.0086 & 0.1172 & 0.2905 & 0.0097 & 0.0240 & 20 \\
\hline Feb. 3,2014 & 10 & 280 & 2.9348 & 0.0040 & 0.0095 & 0.1369 & 0.3224 & 0.0112 & 0.0265 & 4 \\
\hline Mar. 10, 2014 & 10 & 280 & 2.9192 & 0.0047 & 0.0103 & 0.1613 & 0.3528 & 0.0132 & 0.0288 & 5 \\
\hline Apr. 7, 2014 & 10 & 280 & 2.8050 & 0.0040 & 0.0079 & 0.1421 & 0.2808 & 0.0112 & 0.0221 & 13 \\
\hline May 5, 2014 & 10 & 280 & 2.9164 & 0.0035 & 0.0072 & 0.1198 & 0.2480 & 0.0098 & 0.0202 & 9 \\
\hline Jun. 2, 2014 & 10 & 280 & 3.0233 & 0.0046 & 0.0078 & 0.1513 & 0.2596 & 0.0128 & 0.0220 & 9 \\
\hline Jul. 7, 2014 & 10 & 280 & 2.9923 & 0.0047 & 0.0065 & 0.1578 & 0.2157 & 0.0132 & 0.0181 & 19 \\
\hline Aug. 4, 2014 & 10 & 280 & 3.0084 & 0.0040 & 0.0068 & 0.1323 & 0.2269 & 0.0111 & 0.0191 & 20 \\
\hline Sep. 8, 2014 & 10 & 280 & 2.9470 & 0.0038 & 0.0085 & 0.1289 & 0.2889 & 0.0106 & 0.0238 & 14 \\
\hline Oct. 6,2014 & 10 & 280 & 2.9926 & 0.0038 & 0.0083 & 0.1273 & 0.2772 & 0.0107 & 0.0232 & 14 \\
\hline Nov. 3, 2014 & 10 & 280 & 3.0104 & 0.0041 & 0.0070 & 0.1367 & 0.2312 & 0.0115 & 0.0195 & 7 \\
\hline Dec. 8, 2014 & 10 & 280 & 2.9941 & 0.0038 & 0.0068 & 0.1284 & 0.2262 & 0.0108 & 0.0190 & 1 \\
\hline 2014 & 10 & $3,360^{5}$ & 2.958 & 0.0040 & 0.0079 & 0.1367 & 0.2683 & 0.0113 & 0.0222 & $135^{5}$ \\
\hline
\end{tabular}

${ }^{1}$ Mean, standard deviation of repeatability $\left(\mathrm{S}_{\mathrm{r}}\right)$, standard deviation of reproducibility $\left(\mathrm{S}_{\mathrm{R}}\right)$, repeatability limit (r-value), and reproducibility limit (R-value) are expressed on a g/100 g basis. Relative standard deviation of repeatability $\left(\mathrm{RSD}_{\mathrm{r}}\right)$ and relative standard deviation of reproducibility $\left(\mathrm{RSD}_{\mathrm{R}}\right)$ are expressed as a percent.

${ }^{2}$ Year the original collaborative study for the ether extraction method was conducted.

${ }^{3}$ Subsequent years when a proficiency study format was used with milks from 8 different farms to monitor method and laboratory performance. ${ }^{4}$ Method performance conducted over a 4-yr period when a proficiency study format was used with 14 modified milks with an orthogonal design of composition was used to monitor method and laboratory performance.

${ }^{5}$ Total number of tests for the year. 
of how to improve operation. Compared with the collaborative study, it can be seen that within- and between-laboratory agreement improved shortly after the collaborative study, but over several years (i.e., through 1999) not much further improvement in method performance was noted. Starting in 2004, the samples used for the testing were changed to a set of 14 modified milks that was an orthogonal matrix of milk composition, as described above (Kaylegian et al., 2006a). The orthogonal milk sample set made it possible to improve troubleshooting evaluation routines for each analytical method, a process described later in the current paper. The within- and between-laboratory variation in ether extraction results for the modified milk samples from 2007 through 2014 are shown in Table 2 and reflect a decrease $(P<0.05)$ of between 40 to $45 \%$. We hypothesized that this performance improvement was produced by the improved diagnostic and troubleshooting that was made possible by the orthogonal matrix of milk composition. The procedural details of the modified
Mojonnier ether extraction AOAC method (Barbano et al., 1988) have remained unchanged over this complete time period.

Kjeldahl True Protein. The method performance statistics that indicate within- $\left(\mathrm{S}_{\mathrm{r}}\right)$ and betweenlaboratory $\left(\mathrm{S}_{\mathrm{R}}\right)$ variation that were established in the original collaborative study (Barbano et al., 1990) of the determination of total nitrogen content in milk are shown in Table 3. Protein results were expressed on a crude protein basis (i.e., total nitrogen times 6.38). To determine if method performance was changing, the Kjeldahl total nitrogen method was monitored over the period from 1990 to 1995 (Lynch et al., 1997). Each year, 1 or more representatives from each laboratory participating in these studies attended an annual workshop to review performance and focus on details of how to improve operation. Compared with the original collaborative study, the within-laboratory variations in total nitrogen measurement decreased slightly whereas between-laboratory variations increased slightly dur-

Table 3. Method performance statistics ${ }^{1}$ for repeatability and reproducibility by the Kjeldahl total protein and direct true protein method

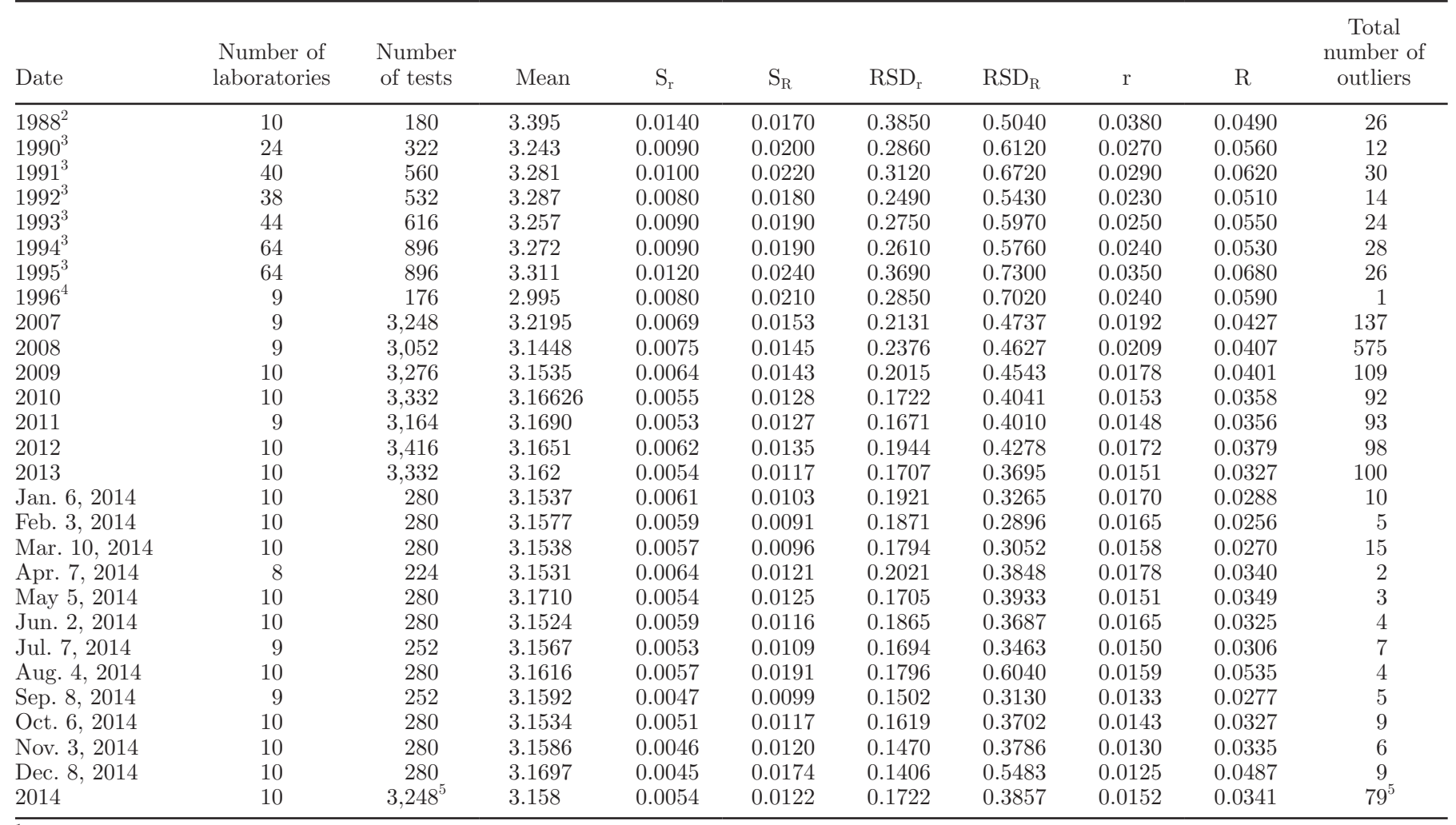

${ }^{1}$ Mean, standard deviation of repeatability $\left(\mathrm{S}_{\mathrm{r}}\right)$, standard deviation of reproducibility $\left(\mathrm{S}_{\mathrm{R}}\right)$, repeatability limit (r-value), and reproducibility limit (R-value) are expressed on a $\mathrm{g} / 100 \mathrm{~g}$ basis. Relative standard deviation of repeatability $\left(\mathrm{RSD}_{\mathrm{r}}\right)$ and relative standard deviation of reproducibility $\left(\mathrm{RSD}_{\mathrm{R}}\right)$ are expressed as a percent.

${ }^{2}$ Original collaborative study results for Kjeldahl total nitrogen method was conducted.

${ }^{3}$ Subsequent years a proficiency study format was used to monitor method and laboratory performance.

${ }^{4}$ Original collaborative study results for direct true protein Kjeldahl method.

${ }^{5}$ Total number of tests for the year. 
Table 4. Method performance statistics ${ }^{1}$ for repeatability and reproducibility by the spectrophotometric enzymatic lactose method

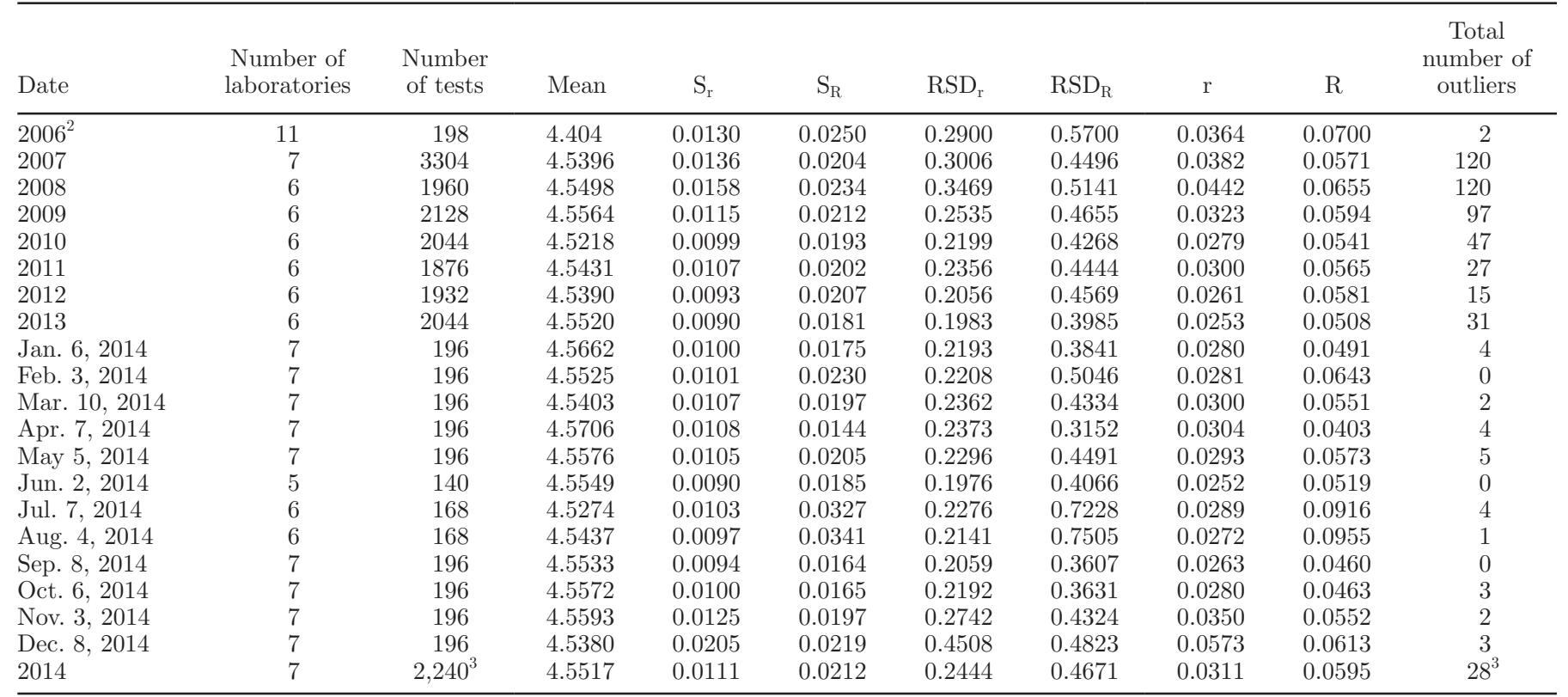

${ }^{1}$ Mean, $\mathrm{S}_{\mathrm{r}}, \mathrm{S}_{\mathrm{R}}$, r, and $\mathrm{R}$ are expressed on a $\mathrm{g} / 100 \mathrm{~g}$ basis. $\mathrm{RSD}_{\mathrm{r}}$ and $\mathrm{RSD}_{\mathrm{R}}$ are expressed as a percent.

${ }^{2}$ Collaborative study results for determination of lactose content of fluid milk using spectrophotometric enzymatic method using weight additions and path length adjustment.

${ }^{3}$ Total number of tests for the year.

Table 5. Method performance statistics ${ }^{1}$ for repeatability and reproducibility of forced-air atmospheric oven-drying method

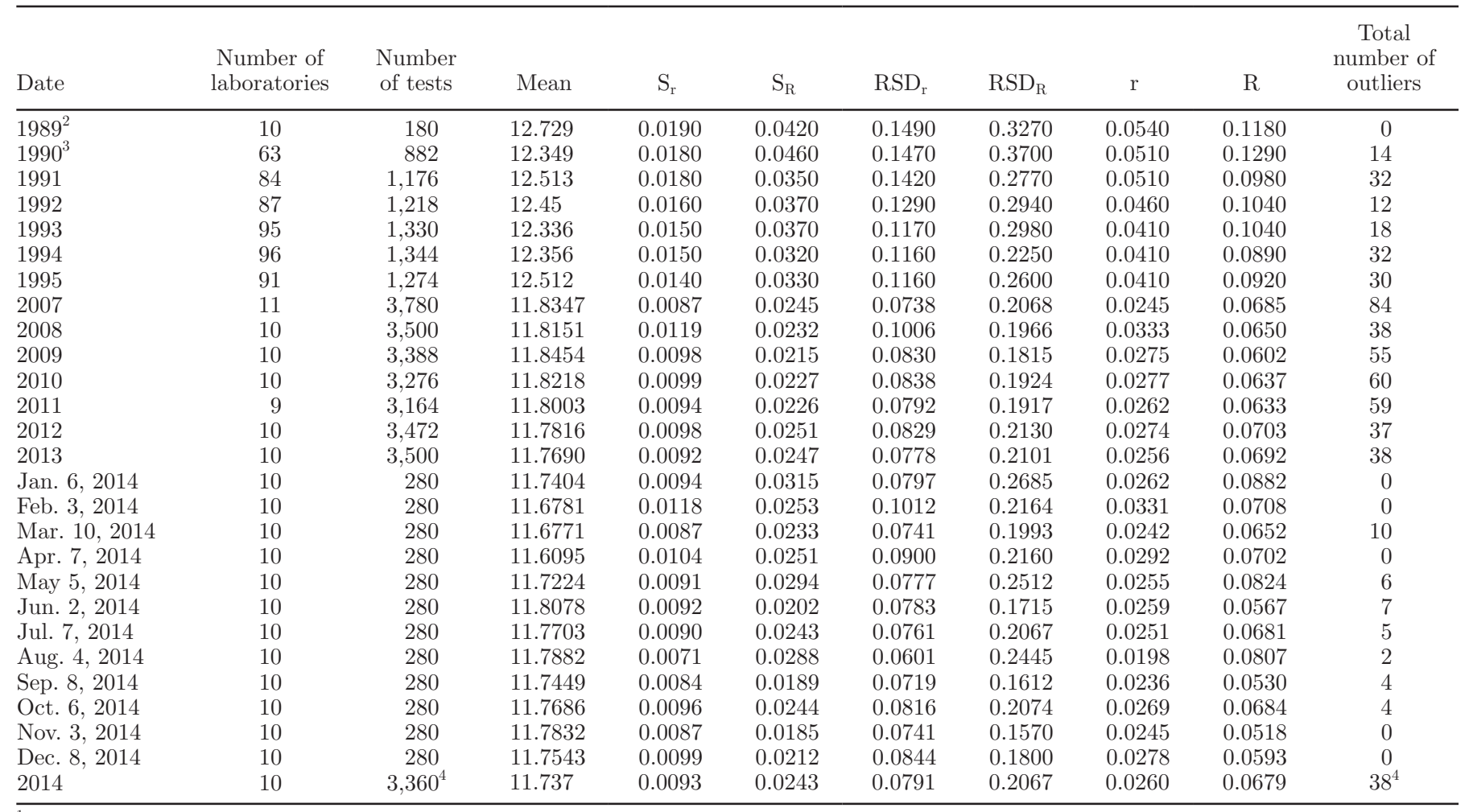

${ }^{1}$ Mean, standard deviation of repeatability $\left(\mathrm{S}_{\mathrm{r}}\right)$, standard deviation of reproducibility $\left(\mathrm{S}_{\mathrm{R}}\right)$, repeatability limit (r-value), and reproducibility limit (R-value) are expressed on a g/100 g basis. Relative standard deviation of repeatability $\left(\mathrm{RSD}_{\mathrm{r}}\right)$ and relative standard deviation of reproducibility $\left(\mathrm{RSD}_{\mathrm{R}}\right)$ are expressed as a percent.

${ }^{2}$ Original collaborative study statistics published in 1989.

${ }^{3}$ Performance evaluation of direct forced-air TS 1990 to 1995.

${ }^{4}$ Total number of tests for the year. 
Table 6. Example of typical Z-scores of participating laboratories within a single year for the ether extraction, Kjeldahl direct true protein, spectrophotometric lactose method, and TS

\begin{tabular}{lcccc}
\hline $\begin{array}{l}\text { Participating } \\
\text { laboratory }\end{array}$ & $\begin{array}{c}\text { Ether } \\
\text { extractions }\end{array}$ & Lactose $^{2}$ & $\begin{array}{c}\text { Kjeldahl direct } \\
\text { true protein }^{1}\end{array}$ & TS $^{1}$ \\
\hline Lab 1 & NA $^{3}$ & 0.9 & 0.6 & 0.3 \\
Lab 2 & 0.2 & 0.5 & 0.4 & 0.4 \\
Lab 3 & 0.6 & 0.4 & 0.9 & 0.3 \\
Lab 4 & 0.6 & NA & 0.5 & 0.4 \\
Lab 5 & 0.8 & NA & 1.4 & 0.4 \\
Lab 6 & 0.3 & 0.7 & 0.3 & 0.6 \\
Lab 7 & 0.5 & 0.4 & 0.5 & 0.4 \\
Lab 8 & 0.5 & 0.8 & 0.3 & 0.2 \\
Lab 9 & 0.4 & NA & 0.5 & 0.7 \\
Lab 10 & 0.5 & NA & 0.7 & 0.7 \\
Lab 11 & 1.1 & 1.1 & 1.0 & 0.9 \\
Mean & 0.6 & 0.7 & 0.6 & 0.5 \\
\hline
\end{tabular}

${ }^{1}$ Laboratory data from March 9, 2009.

${ }^{2}$ Laboratory data from June 8, 2009.

${ }^{3} \mathrm{NA}=$ not applicable. The laboratory did not run this test.

ing the years 1990 to 1995. In 1991, a collaborative study was conducted to develop new direct and indirect Kjeldahl methods of the measurement of true protein content of whole milk, because about $6 \%$ of the total nitrogen content of milk is nonprotein nitro- gen. Total nitrogen content of milk multiplied by the conversion factor 6.38 overestimates the true protein content of milk on average by about $6 \%$ (Barbano et al., 1991). The direct true protein method became the basis for milk payment testing in the USDA Federal Milk Markets in the United States in the year 2000 (USDA, 2000). In Table 3, the method performance statistics for the years 2007 through 2014 are based on the Kjeldahl true protein method. The mean betweenlaboratory performance statistics were significantly $(P$ $<0.05$ ) better from 2007 to 2014 (i.e., $\mathrm{S}_{\mathrm{R}}, \mathrm{RSD}_{\mathrm{R}}, \mathrm{R}$ ) than in the collaborative study (Barbano et al., 1991).

Enzymatic Lactose. The indices of within- $\left(\mathrm{S}_{\mathrm{r}}\right)$ and between-laboratory $\left(\mathrm{S}_{\mathrm{R}}\right)$ method performance from the original collaborative study (Lynch et al., 2007) and all results for the subsequent years (2007 through 2014) are found in Table 4 . We observed no significant $(P>$ 0.05 ) changes in within- or between-laboratory method performance over this period of time.

Forced-Air, Oven-Drying TS. The original collaborative study was conducted in 1989 and the method performance statistics are found in Table 5. Additional method performance statistics for the period from 1990 to 1995 are also provided. During the years 1990 to

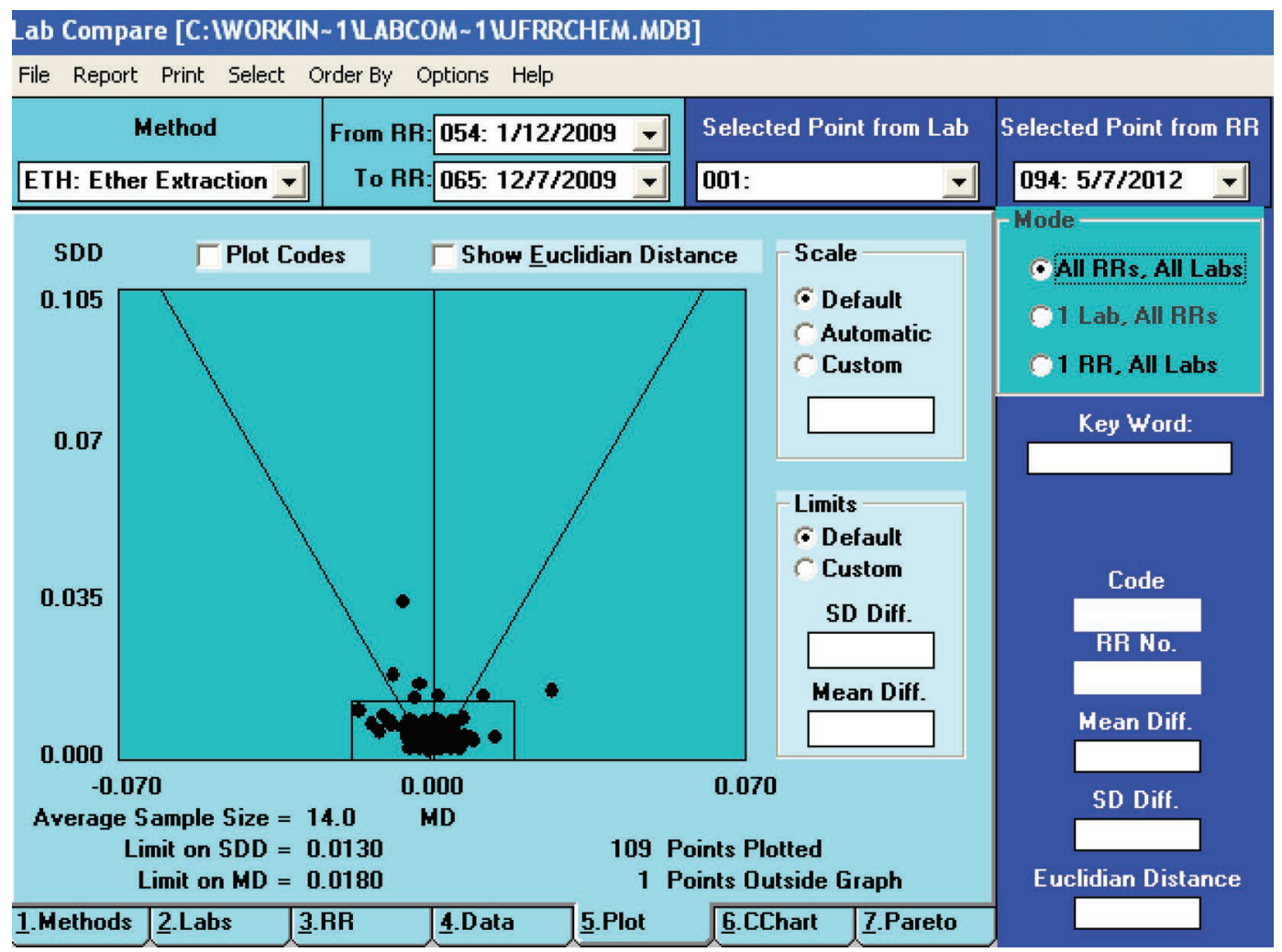

Figure 1. Euclidean distance plot of ether extraction method (AOAC Method 989.05; AOAC International, 2000) variation among laboratories in 2009. Software: Lab Compare V1.30, Cornell University, Ithaca, NY. RR = round robin; SDD = standard deviation of the difference. Color version available online. 
Table 7. Z-score ranges and total Z-score relative percentages of Z-scores within each range for ether extraction, Kjeldahl direct true protein, lactose, and TS for laboratories that participated over the 3-yr period from 2007 through 2009

\begin{tabular}{lrrrr}
\hline Z-score range & $\begin{array}{c}\text { Ether } \\
\text { extraction }\end{array}$ & Kjeldahl direct & & \\
true protein $^{2}(\%)$ & Lactose $^{3}(\%)$ & TS $^{4}(\%)$ \\
\hline 0.0 to $<0.5$ & 62.8 & 37.6 & 38.2 & 61.5 \\
0.5 to $<1.0$ & 29.6 & 49.4 & 42.5 & 34.3 \\
1.0 to $<1.5$ & 4.0 & 8.4 & 15.5 & 2.1 \\
1.5 to $<2.0$ & 2.1 & 2.0 & 2.1 & 1.3 \\
2.0 to $<2.5$ & 0.5 & 0.0 & 0.9 & 0.0 \\
2.5 to $<3.0$ & 0.0 & 0.6 & 0.4 & 0.5 \\
3.0 to $<3.5$ & 0.0 & 0.3 & 0.4 & 0.0 \\
3.5 to $<4.0$ & 0.3 & 0.0 & 0.0 & 0.0 \\
4.0 to $<4.5$ & 0.0 & 0.6 & 0.0 & 0.3 \\
4.5 to $<5.0$ & 0.0 & 1.1 & 0.0 & 0.0 \\
7.5 to $<8.0$ & 0.3 & 0.0 & 0.0 & 95.8 \\
Z-score percentage $<1.0$ & 92.4 & 87.0 & 80.7 & \\
\hline
\end{tabular}

${ }^{1}$ Based on an average of 10 labs participating per month per year (374 total Z-scores).

${ }^{2}$ Based on an average of 9 labs participating per month per year (356 total Z-scores).

${ }^{3}$ Based on an average of 7 labs participating per month per year (233 total Z-scores).

${ }^{4}$ Based on an average of 10 labs participating per month per year (382 total Z-scores).

1995, both the within- and between-laboratory method performance improved relative to the collaborative study. However, the data for the period 2007 through 2014 reflect a much larger improvement $(P<0.05)$, particularly for between laboratory performance (i.e., $\mathrm{S}_{\mathrm{R}}$ ). This large improvement in between-laboratory agreement is related to the improved ability to identify and troubleshoot between-laboratory differences when

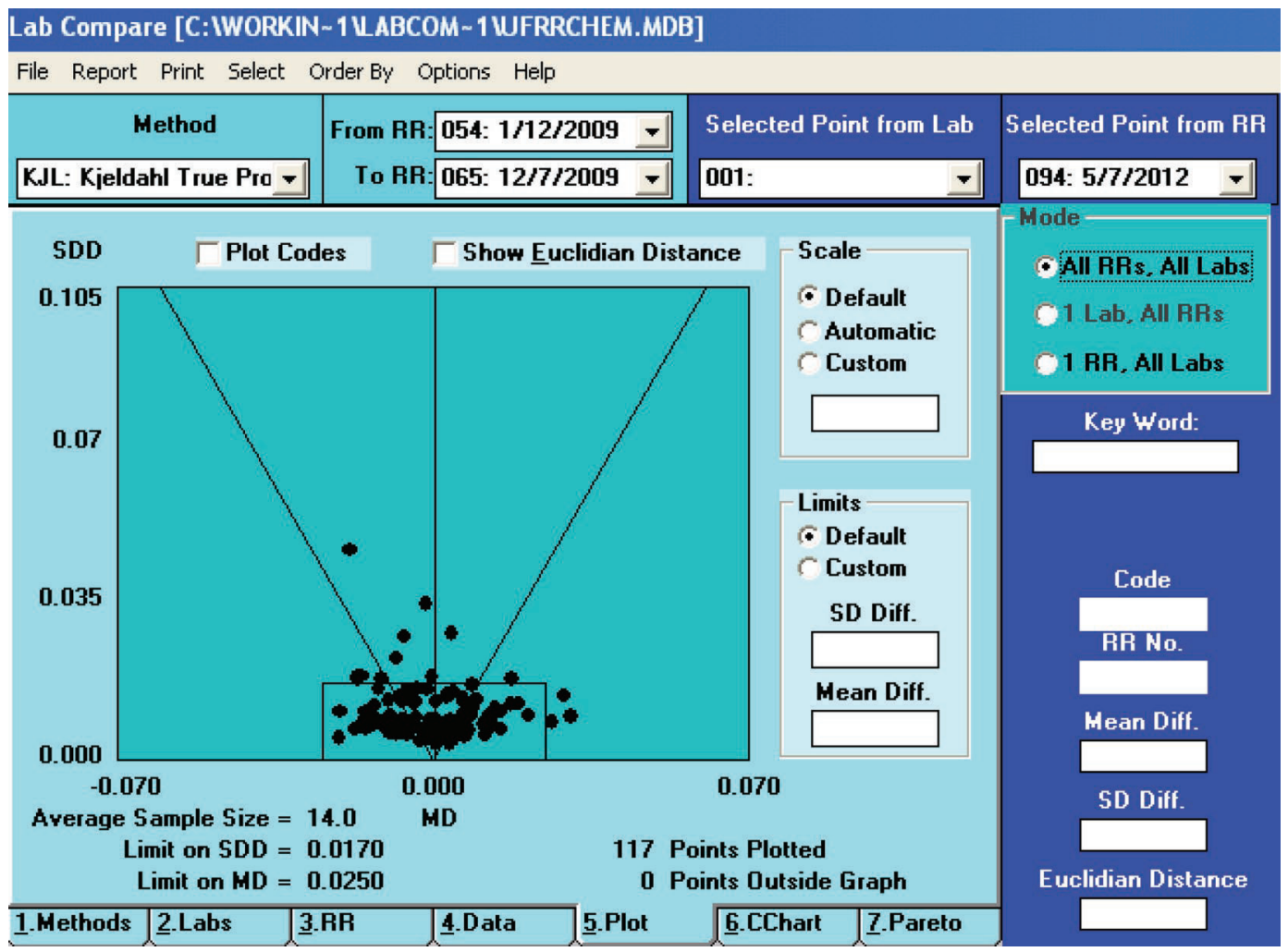

Figure 2. Euclidean distance plot of Kjeldahl true protein method variation (AOAC method 991.22; AOAC International, 2000) among laboratories in 2009. Software: Lab Compare V1.30, Cornell University, Ithaca, NY. RR = round robin; SDD = standard deviation of the difference. Color version available online. 
using the modified milks with the orthogonal design of variation in composition. Examples of the troubleshooting to identify and correct method performance will be provided in the next sections.

\section{Proficiency Testing}

Z-Scores. In Table 6, 2 representative months in 2009 were chosen to give an overall view of the typical Z-scores for each laboratory participating in the collaborative study. In our study, Z-scores were calculated for 2007, 2008, and 2009 data for each method and the results are shown in Table 7 . For all 3 yr, $92.4 \%$ of ether extraction Z-scores were $<1.0,87 \%$ of Kjeldahl Zscores were $<1.0,80.7 \%$ of lactose Z-scores were $<1.0$, and $95.8 \%$ of TS Z-scores were $<1.0$. The proportion of Z-scores $<1$ was much higher than would be expected and the proportion $>3$ was lower than expected based on Gaussian distribution (Table 7). If the long-term reproducibility value $\left(\mathrm{S}_{\mathrm{R}}\right)$ used in the Z-score calculation was representative of the current reproducibility of the method, then about $5 \%$ of the Z-scores should be $>3$. This was not observed because, mathematically, the mean Z-score continued to become smaller and smaller as a group laboratory improved their method performance because the numerator in the equation (sample replicate - population mean/long-term $S_{R}$ value) became smaller over time. Again, the denominator is the $S_{R}$ value from the original collaborative study. Because the $S_{R}$ value remained constant in the calculation over time, but the actual $S_{R}$ for the population improved with time after the collaborative study (which it did; see Tables 2, 3, 4, 5), the mean Z-score and the laboratory-to-laboratory variation in Z-score decreased. As this happens, the Z-scores become less meaningful as a true gauge of between-laboratory performance. To maintain and improve the meaning of the Z-score in a proficiency-testing scheme, changing the long-term $S_{R}$ used in Z-score calculation to a value that is based on the annual mean $S_{R}$ observed in the proficiency testing would be an approach to maintain the benefit of the Z-score system across time as the method performance for a group of laboratories improves (i.e., $\mathrm{S}_{\mathrm{R}}$ decreases) after a collaborative study.

ED Plots. Figures 1 through 4 are example ED plots for fat, protein, lactose, and TS, respectively, in data

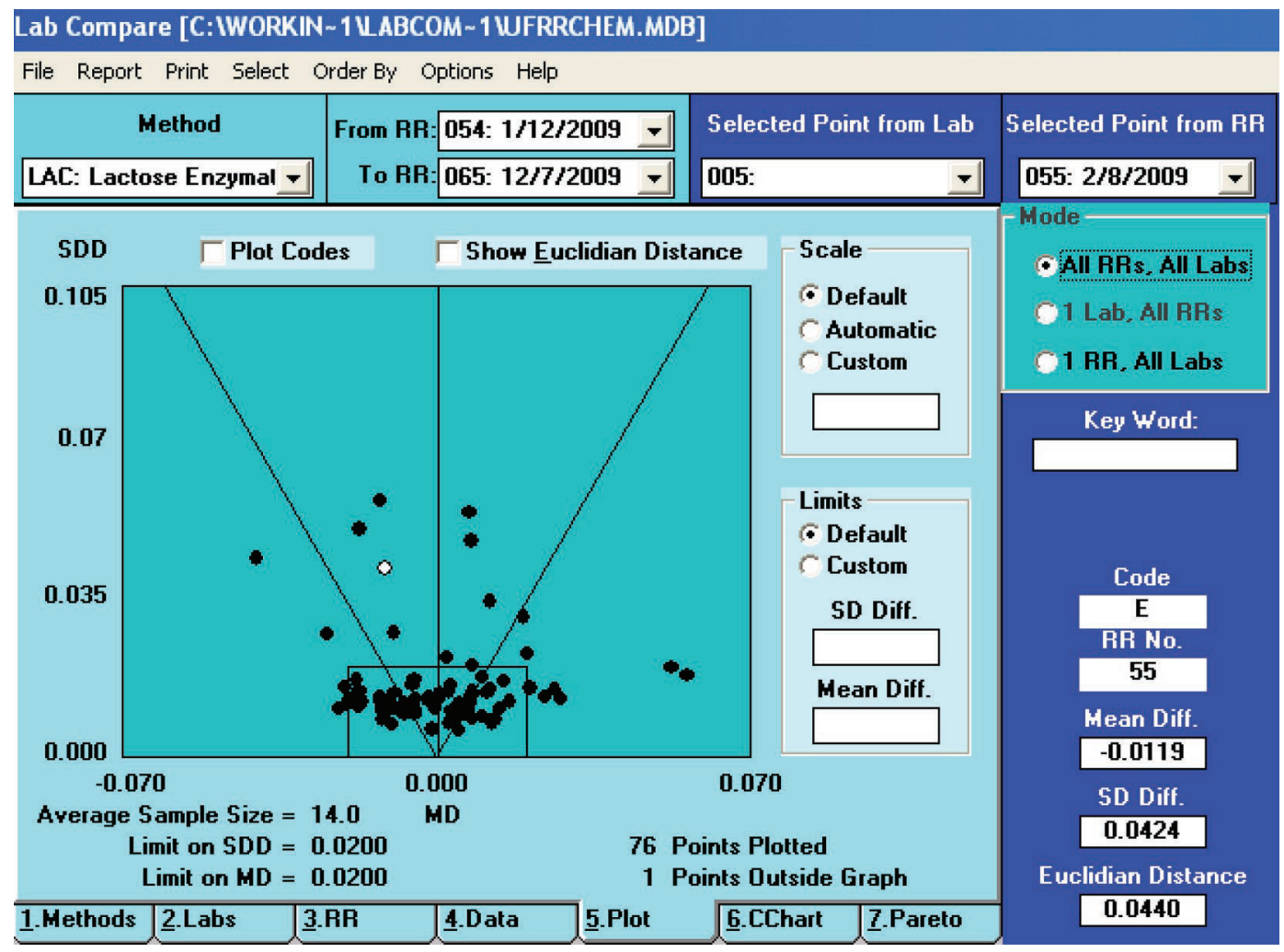

Figure 3. Euclidean distance plot of enzymatic lactose method variation (AOAC method 2006.06; AOAC International, 2000) among laboratories in 2009. Software: Lab Compare V1.30, Cornell University, Ithaca, NY. RR = round robin; SDD = standard deviation of the difference. Color version available online. 
analysis software developed for the quality-assurance program using a Microsoft Access database (Microsoft Corp., Redmond, WA). The testing method, proficiency test round robin (i.e., date of sample exchange), and the laboratory identification can be displayed for a group of laboratories over a selected period of time (e.g., last month, last year, last $n$ years, and so on). Once these parameters are selected, all the graphic views of the data can be presented for the selected method and period of time for one or all laboratories. The tabs along the bottom of the screen allow selection or addition of new methods, new laboratories, added sample exchanges, data input for each sample exchange, Euclidean plots, control charts, and Pareto diagrams. Default limits for axes and chart limits can be set in the various pull-down menus. Data output can be viewed or printed with or without laboratory identification or in blind coded format. Euclidean distance plots can be presented for the full group of laboratories for any segment of time or for 1 laboratory across a selected period of time. The MD and SDD limits on the box on in the center of the plot are based on the method performance in the collaborative study of each method.
The box limits represents the zone in which laboratory data should reside $95 \%$ of the time.

Figures 1 and 2 represent data for fat determined by ether extraction and true protein determined by Kjeldahl. These figures indicate that both methods are performing well in the group of laboratories relative to the expected performance based on the collaborative study method statistics because $>95 \%$ of the points are within the box. When the laboratory identifications are revealed for individual data points (not shown), it becomes apparent in Figure 2 that the high SDD are all from 1 laboratory and troubleshooting follow up by the proficiency testing coordinator with that laboratory is appropriate, using procedures recommended in the Kjeldahl troubleshooting procedures described by Lynch and Barbano (1999). In Figure 3 for the lactose method, it many data points can be observed indicating high SDD for some laboratories. When this changes from one period of time to another, demonstrating unexpectedly high variation that is not systematic, it may reflect a problem with something in the method. In the case of spectrophotometric lactose analysis, we have experienced this behavior when a change in the

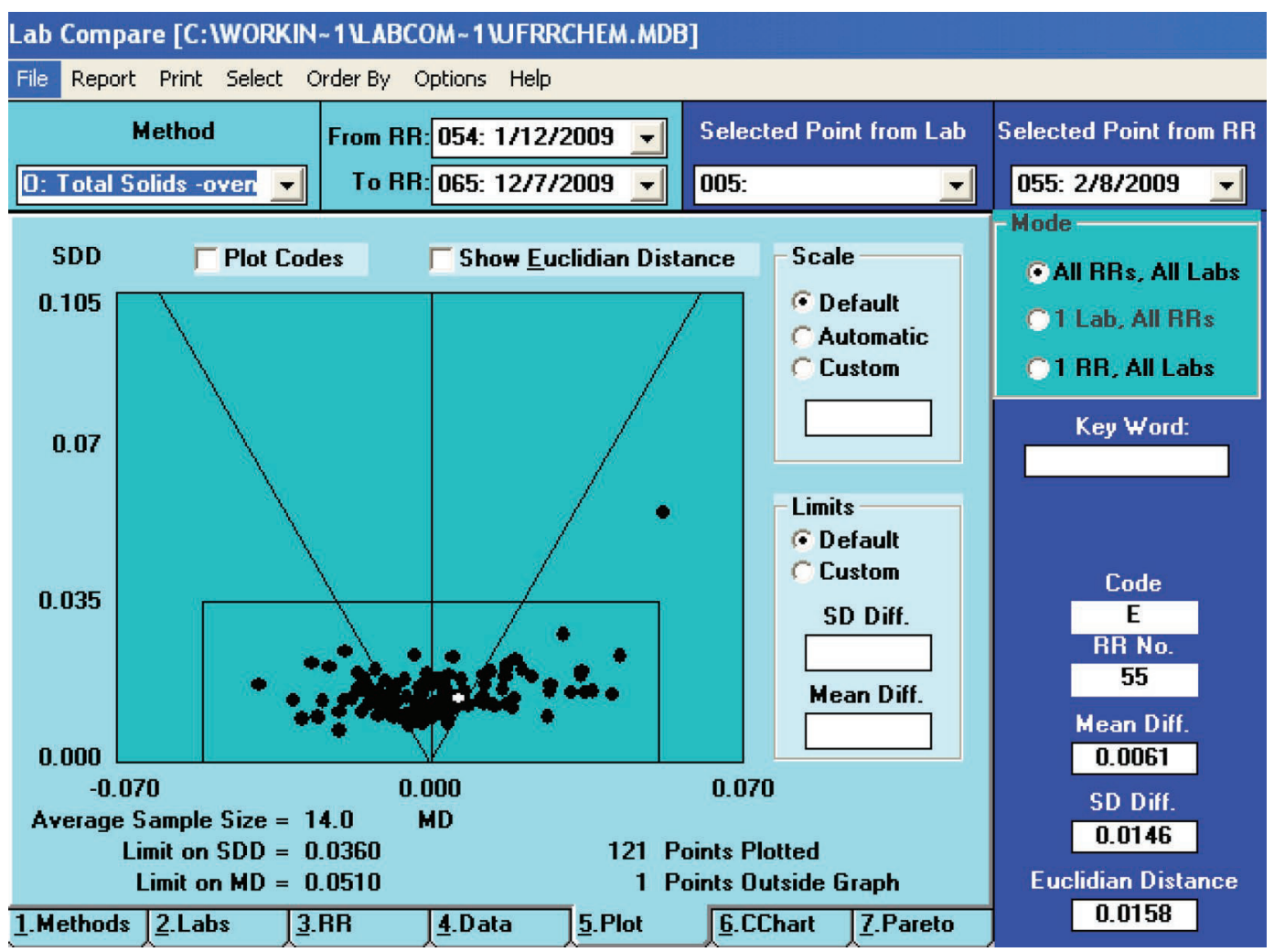

Figure 4. Euclidean distance plot of oven-drying TS method variation (AOAC method 990.20; AOAC International, 2000) among laboratories in 2009. Software: Lab Compare V1.30, Cornell University, Ithaca, NY. RR = round robin; SDD = standard deviation of the difference. Color version available online. 
quality of disposable cuvettes and their lids occurs that leads to leakage during mixing in the assay. In this case, the quality-assurance program can identify when action is needed to interface with an equipment or reagent provider for a method. Figure 4 for forced-air, oven-drying TS represents a method that is performing better or consistent with the expectation based on the collaborative study of the same method. Control charts for each laboratory to show changes in MD and SDD across time with statistical control limits can be displayed (not shown) and used to help an individual laboratory identify when performance is changing or unacceptable and identify the factors that are influencing the analytical performance of each chemical method in their laboratory

Pareto Diagrams. Figures 5 through 8 are examples Pareto diagrams (of ED) for fat, protein, lactose, and TS, respectively, in data analysis software developed for the quality-assurance program using a Microsoft Access database (Microsoft Corp.). In this case, a Pareto diagram for MD, SDD, or ED for a single sample exchange or sample exchanges across a selected period of time can be displayed to rank the performance of all labora- tories on each parameter. This allows each laboratory to compare their performance to other laboratories and for a laboratory to determine if their performance is changing relative to other laboratories in the group. Key words (e.g., name of analyst, brand of equipment, and so on) can be used to further parse data to identify factors influencing the method performance within or between laboratories. The Pareto diagrams are useful to help staff responsible for quality-assurance across laboratories to quickly identify the laboratories where technical troubleshooting support is needed. In Figures 5,7 , and 8 , it is clear that 1 laboratory in each method has a greater need for troubleshooting help and possibly method training review if recent turnover of analytical laboratory staff has occurred or if something is malfunctioning with equipment for that method. The Kjeldahl data in Figure 6 shows no extreme laboratories with respect to performance problems. In this case, the mean performance for the best $50 \%$ of the laboratories becomes the achievable performance goal for the lowerperforming laboratories. The best-performing laboratories and analysts are selected from the group to make presentations of their experience and advice on the

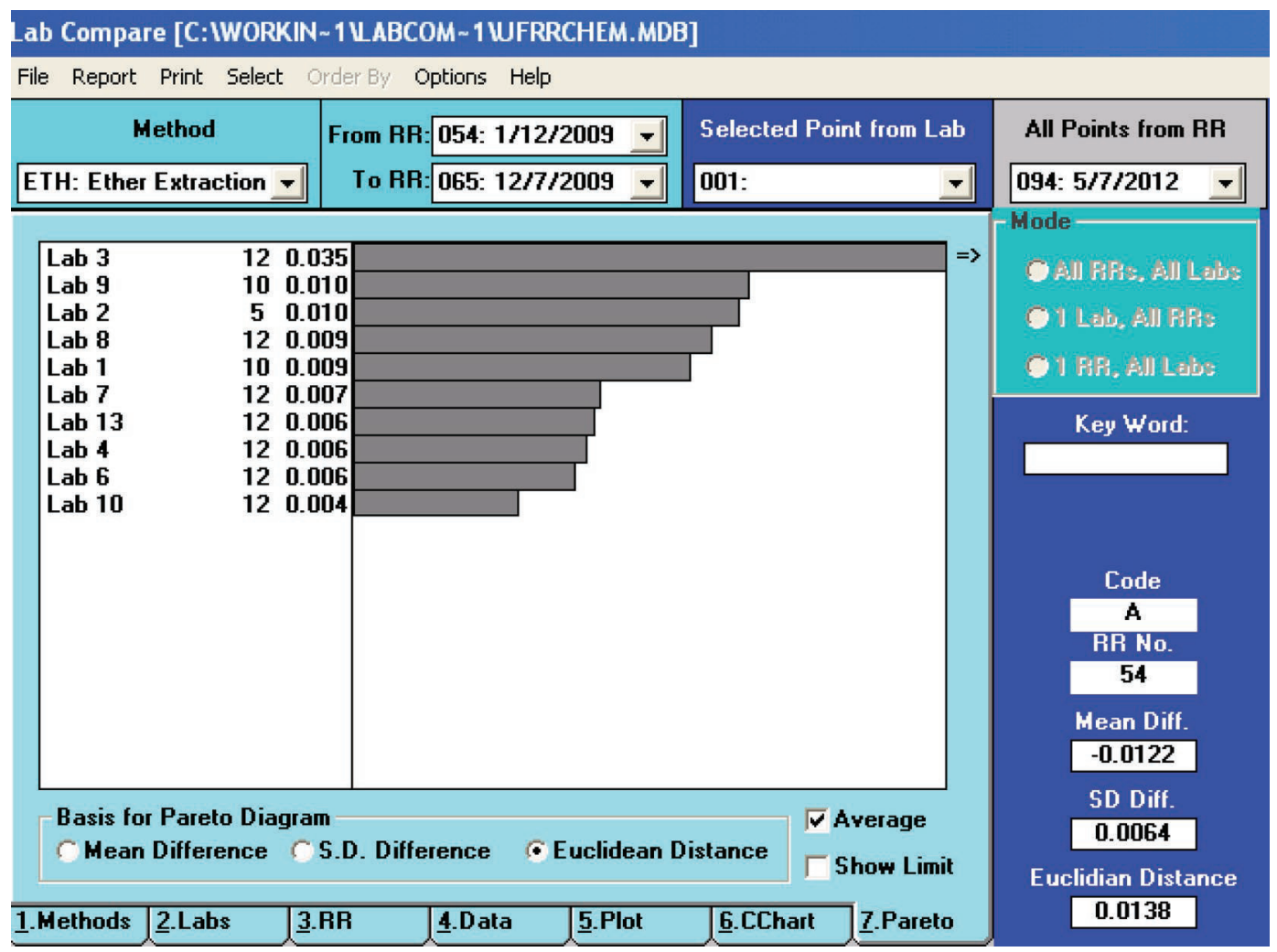

Figure 5. Pareto diagram of ether extraction performance (AOAC method 989.05; AOAC International, 2000) among laboratories in 2009. Software: Lab Compare V1.30, Cornell University, Ithaca, NY. RR = round robin; SDD = standard deviation of the difference. Color version available online. 
method at the annual quality-assurance and training laboratory workshop for this network of laboratories. The sharing of the experience, questions, and answers promotes collaboration among the laboratories as they try to achieve better performance as a group.

\section{Examples of Within-Laboratory Troubleshooting}

Ether Extraction. The next step in the qualityassurance process is to focus more specifically at the analyst level within each method and each monthly exchange of milk samples. Figures 9, 10, and 11 are examples of graphs of the residual difference data [test value of the laboratory minus the all-laboratory mean (outliers excluded)] for each sample for 1 analyst on 1 set of the 14 milks (described above) tested in duplicate plotted as a function of fat concentration in the milk using Microsoft Excel Office 2010 (Microsoft Corp.). The design of the 14-sample set with a wide range of equally spaced concentrations increases the power of this step in method performance troubleshooting. Over the many years of doing this, we developed the experience to relate the behavior of residual plots to specific systematic and random sources of error in the performance of each of these methods that have such great effect on milk payment testing. In Figure 9, it is clear that a systematic (high $\mathrm{R}^{2}$ of the slope of the residual plot) error has occurred for fat measurement in this laboratory on this set of milks. In our experience, the possible causes are water level in the water bath too low when tempering the milk samples before milk mixing and weighing at the start of the analysis, last samples (high fat) not full up to temperature before mixing or fat stuck on the inside of the lid of the container, or inadequate sample mixing by inversion on the high-fat samples (i.e., scatter of points more at high fat levels). In Figure 10, the source of deviation from the all-laboratory mean is different than in Figure 9, but it is systematic, as indicated by the high coefficient of determination of the regression equation. In this case, our experience is that a problem exists in the final drying step of the fat residue in the fat-collection vessel. For higher-fat milks, the amount of extract fat in the container is larger (i.e., deeper layer of liquid fat) and it is harder to remove the last of residual moisture during the drying step on higher-fat milks. The other possibil-

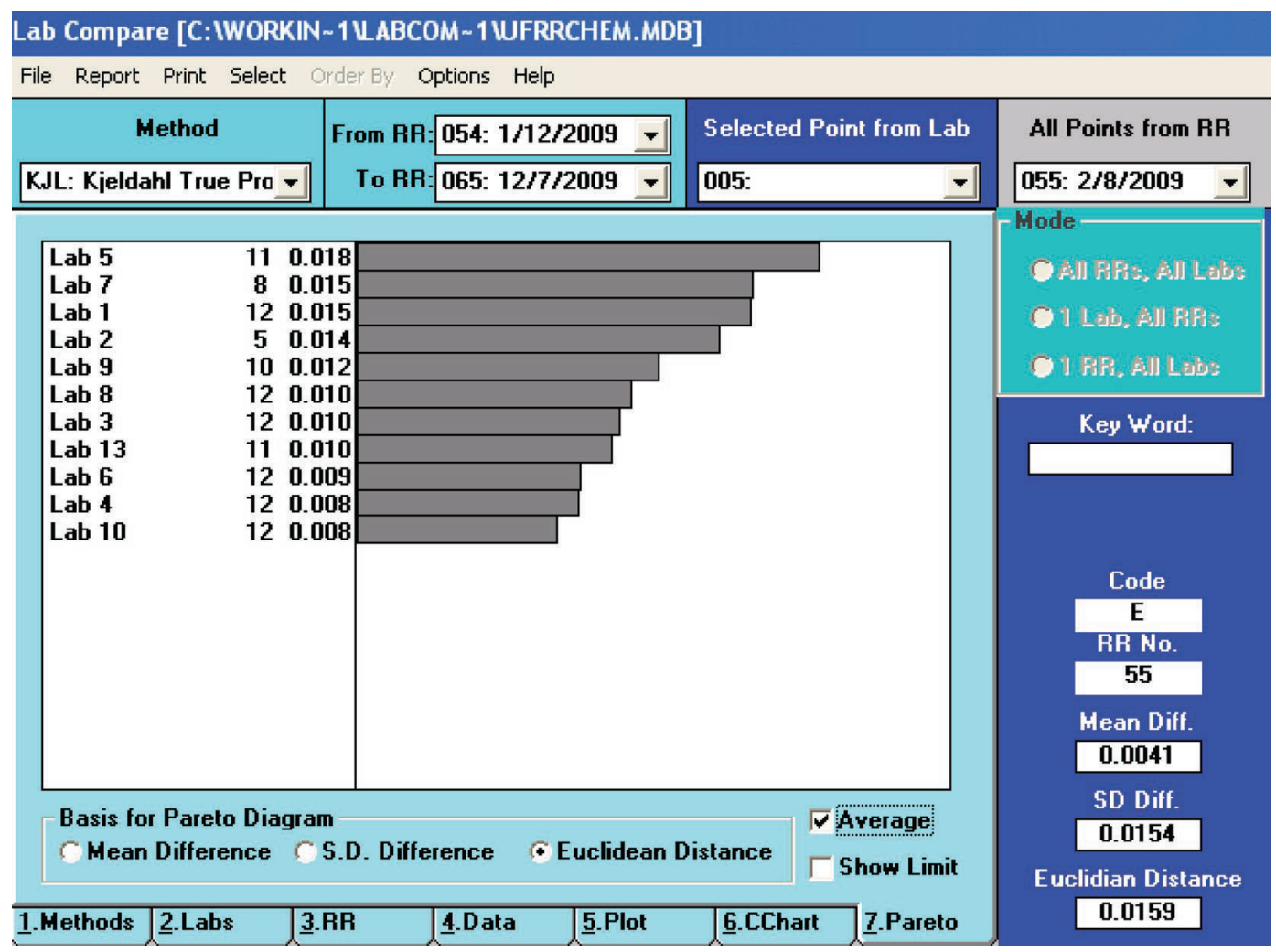

Figure 6. Pareto diagram of Kjeldahl true protein method variation (AOAC method 991.22; AOAC International, 2000) among laboratories in 2009. Software: Lab Compare V1.30, Cornell University, Ithaca, NY. RR = round robin; SDD = standard deviation of the difference. Color version available online. 
ity is something has happened to the oven temperature and it is too low, or perhaps the analyst was in a hurry at the end of the day and did not leave the samples in the oven for the full length of time. The data in Figure 11, represents the performance of the Mojonnier method and a skilled analyst over the range of fat content of milk from 0.2 to nearly $6 \%$ fat. The best and most experienced analysts hardly ever have a residual difference of more than $0.01 \%$ across the full range of fat content in milks for producer milk testing.

Kjeldahl. Figures 12 through 15 are examples of graphs of the residual difference data (test value of the laboratory minus the all-laboratory mean with outliers excluded) for each sample for one analyst on one set of the 14 milks tested in duplicate (described above) plotted as a function of true protein concentration in the milk. The data in Figure 12 is from a laboratory that uses a 12 over 12 position traditional Kjeldahl digestion and distillation system. An up and down pattern of alternating differences is noticeable in the residual difference for the first 11 or 12 milks. In general, the milks with the lower residual differences were run together as one set of digestion and distillations and the milks with the higher residual differences were run as a separate set of digestion and distillations. Two samples and the blanks were run as a third partial set of samples (28 flasks plus 2 blanks). This pattern shows up in the residual plots for data collected on a traditional system in some laboratories that use cold tap water for cooling during the distillation. This pattern in the data is due to either the water temperature of distillation cooling water being different between the distillation sets or a difference in the temperature of the boric acid collection solution at the beginning the distillation. This behavior is more common in summer months when the distillation cooling water is warmer than in winter months.

The residual plot in Figure 13 shows a consistent bias across the full concentration range of protein, which is caused either by consistently low nitrogen recovery during digestion or a systematic error in the blank value. If the acid digests cannot be cooled to room temperature without crystallization before water addition for distillation, it is a clear indication of a problem of too little residual acid at the end of digestion. Procedures for troubleshooting and correcting low nitrogen recovery are discussed by Lynch and Barbano (1999). A consis-

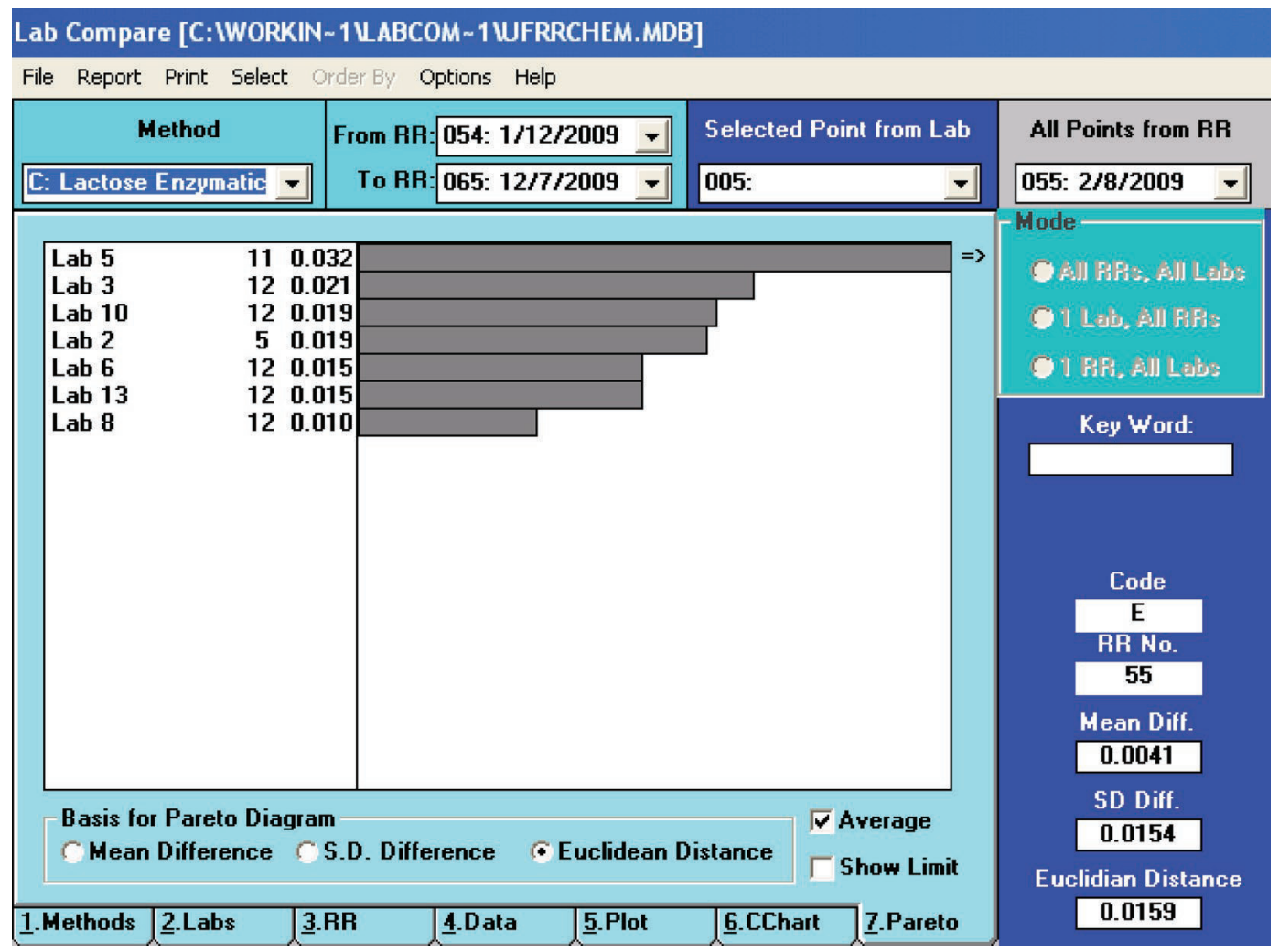

Figure 7. Pareto diagram of enzymatic lactose method variation (AOAC method 2006.06; AOAC International, 2000) among laboratories in 2009. Software: Lab Compare V1.30, Cornell University, Ithaca, NY. RR = round robin; SDD = standard deviation of the difference. Color version available online. 


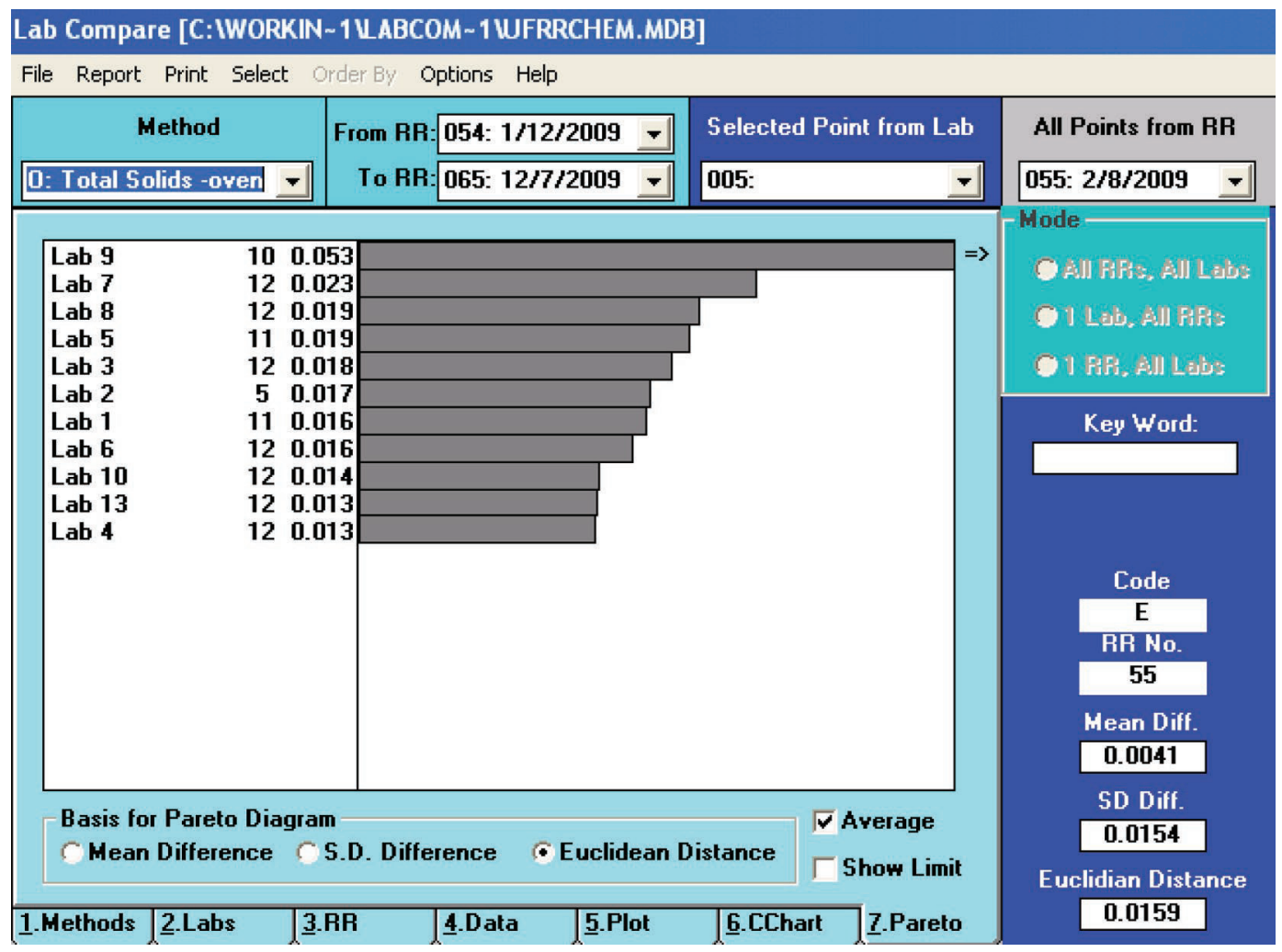

Figure 8. Pareto diagram of oven-drying TS method variation (AOAC 990.20; AOAC International, 2000) among laboratories in 2009. Software: Lab Compare V1.30, Cornell University, Ithaca, NY. RR = round robin; SDD = standard deviation of the difference. Color version available online.

tent high bias (data not shown) can occur, which may be caused by extremely low blank values (or negative blanks). Negative blanks (zero titration volume) can be caused by contamination of the boric acid solution or the distillation collection flasks. Numerous other factors can cause a systematic high bias, including a systematic error in the software for calculation of the results.

Another behavior of data we commonly observe in residual plots from laboratory to laboratory is shown in

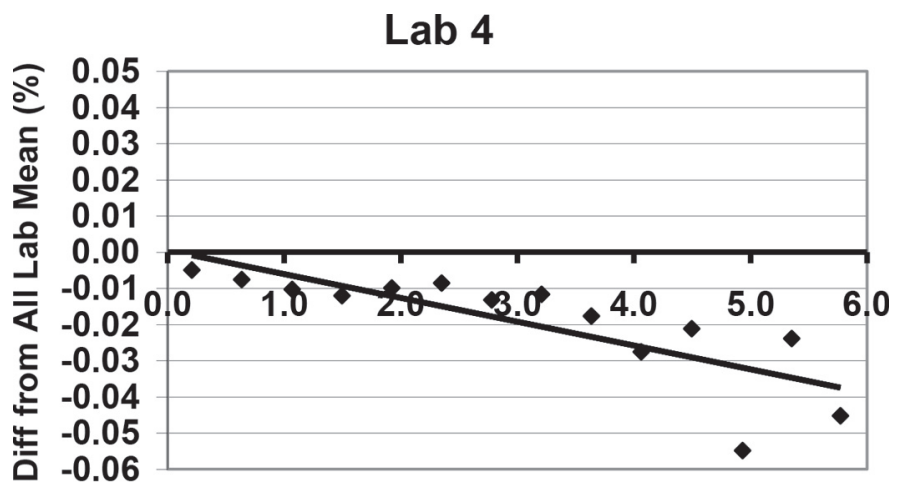

$$
\begin{array}{cc}
y=-0.0066 x+0.0005 & \text { Fat }(\%) \\
R^{2}=0.6438 &
\end{array}
$$

Figure 9. Residual plot of differences from the all-laboratory mean ether extraction (AOAC method 989.05; AOAC International, 2000) result for laboratory 4 , as a function of fat content of milk.

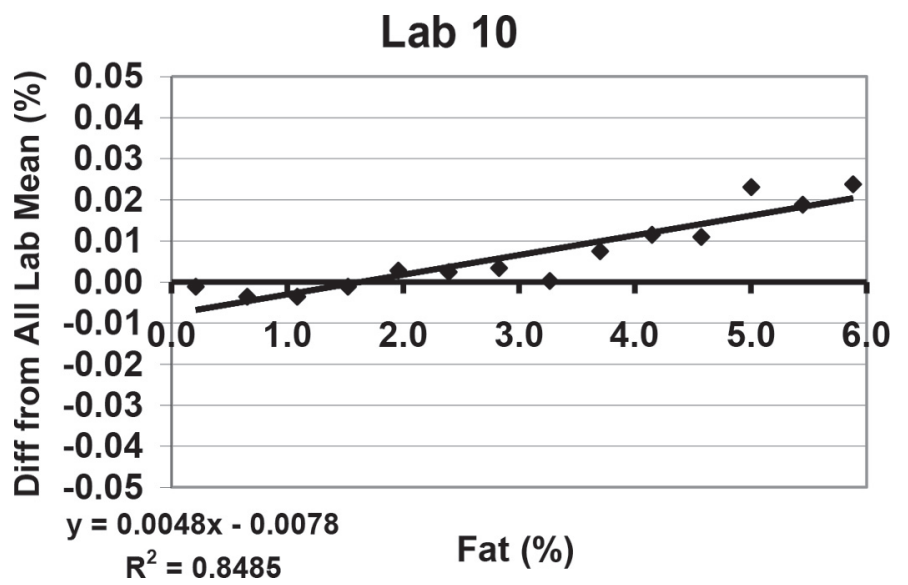

Figure 10. Residual plot of differences from the all-laboratory mean ether extraction (AOAC method 989.05; AOAC International, 2000) result for laboratory 10 , as a function of fat content of milk. 


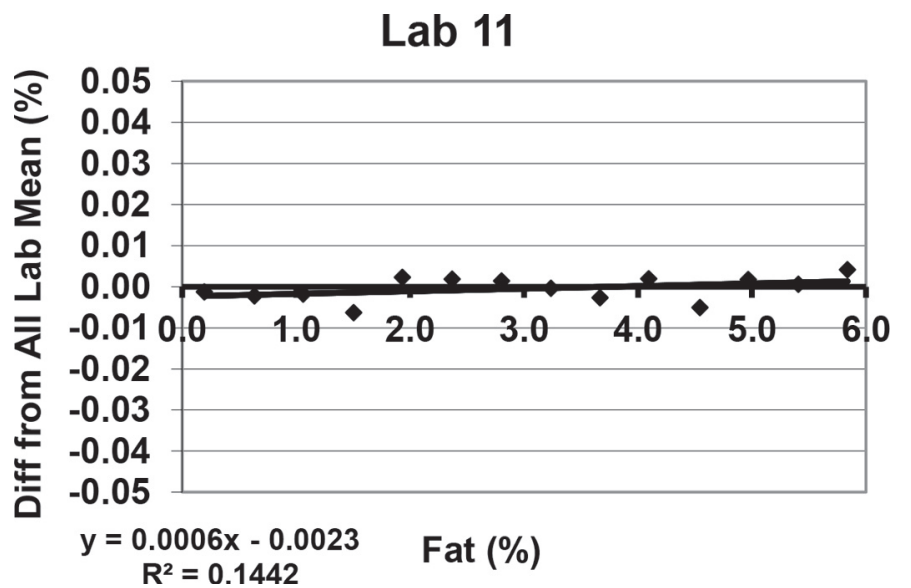

Figure 11. Residual plot of differences from the all-laboratory mean ether extraction (AOAC method 989.05; AOAC International, 2000) result for laboratory 11 , as a function of fat content of milk.

Figures 14 and 15. The high coefficient of determination indicates that the cause is systematic and possible to identify. Over the years, we have developed an understanding of the cause of this behavior of residual difference data from laboratory to laboratory. It is related to the accuracy of the normality of the solution actually being used to carry out the titration in a laboratory. The official methods for both $\mathrm{CP}$ determination (Barbano et al., 1990) and for true protein (Barbano et al., 1991) both require a titrant $\mathrm{HCl}$ normality of $0.1000 \pm 0.0005$. Typically, laboratories purchase prestandardized $\mathrm{HCl}$ sold with this specification. Often the reagent comes with a certificate of analysis for the lot of $\mathrm{HCl}$. It has been our experience that lots of $\mathrm{HCl}$ are sometimes out of specification and their normality does not agree with the certificate of analysis. This is a very

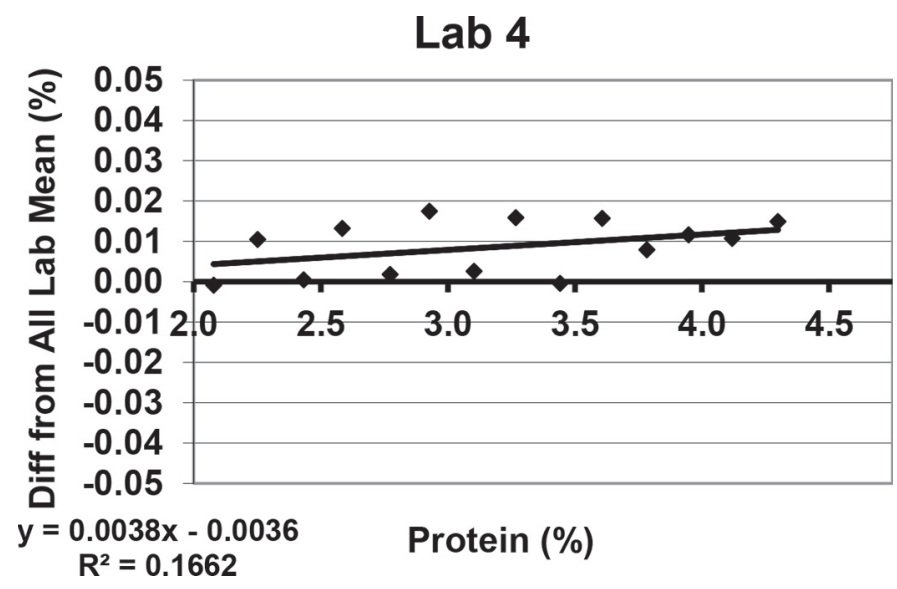

Figure 12. Residual plot of differences from the all-laboratory mean Kjeldahl true protein (AOAC method 991.22; AOAC International, 2000) result for laboratory 4 , as a function of true protein content of milk.

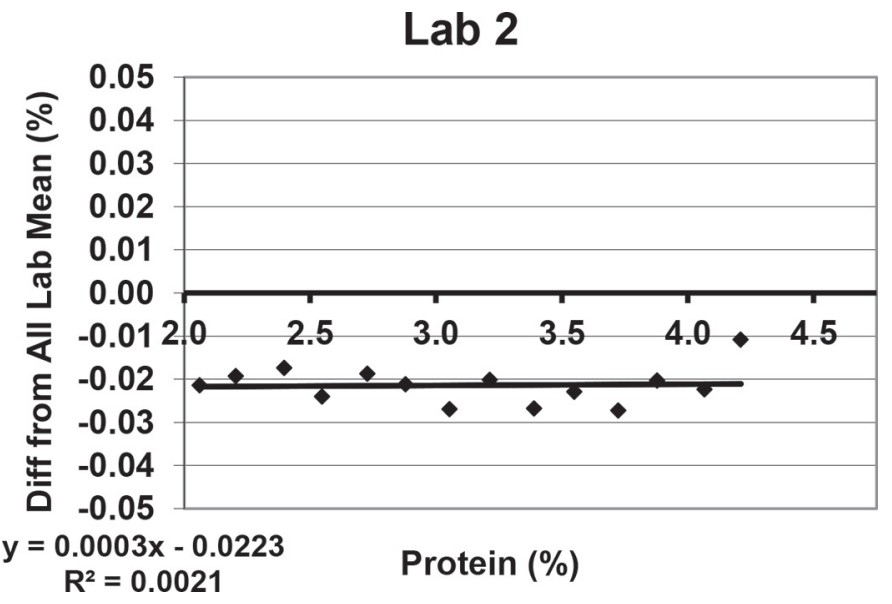

Figure 13. Residual plot of differences from the all-laboratory mean Kjeldahl true protein (AOAC method 991.22; AOAC International, 2000) result for laboratory 2 , as a function of true protein content of milk.

challenging issue and has resulted in development of inhouse relative normality-checking procedures (based on weights in titration) to check the difference in normality between a current and new batch of $0.1000 \mathrm{~N} \mathrm{HCl}$ to compare the relative difference in the certificate of analyses of the 2 batches. The decision for a laboratory is whether to use either the nominal value for normality of $0.1000 \mathrm{~N}$ in their calculation or to use the value from the certificate of analysis. The reality is that the value on the certificate of analysis has some degree of uncertainty (usually not given) and that the behavior of data for a laboratory in the proficiency analysis and the relative determination of the new batch of titrant may indicate that actual batch of reagent being used does

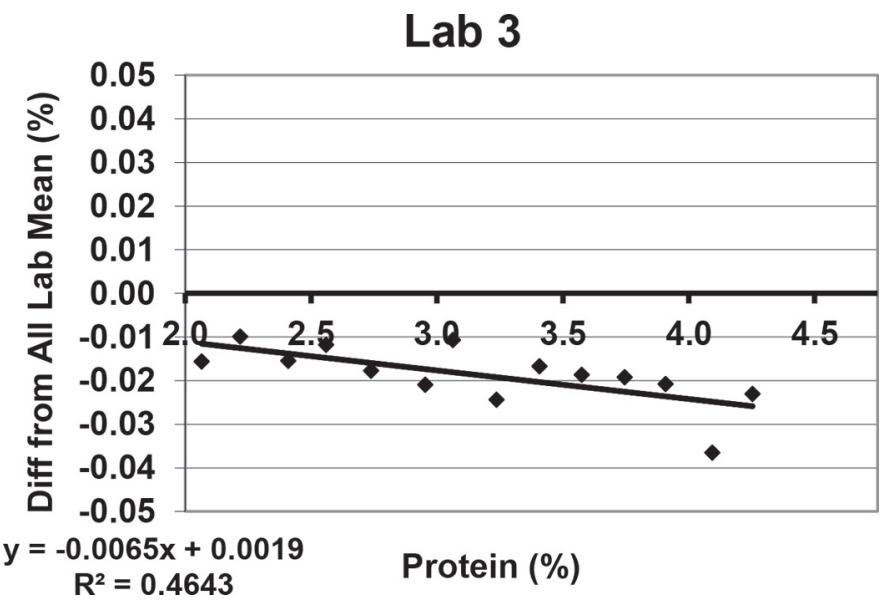

Figure 14. Residual plot of differences from the all-laboratory mean Kjeldahl true protein (AOAC method 991.22; AOAC International, 2000) result for laboratory 3 , as a function of true protein content of milk. 


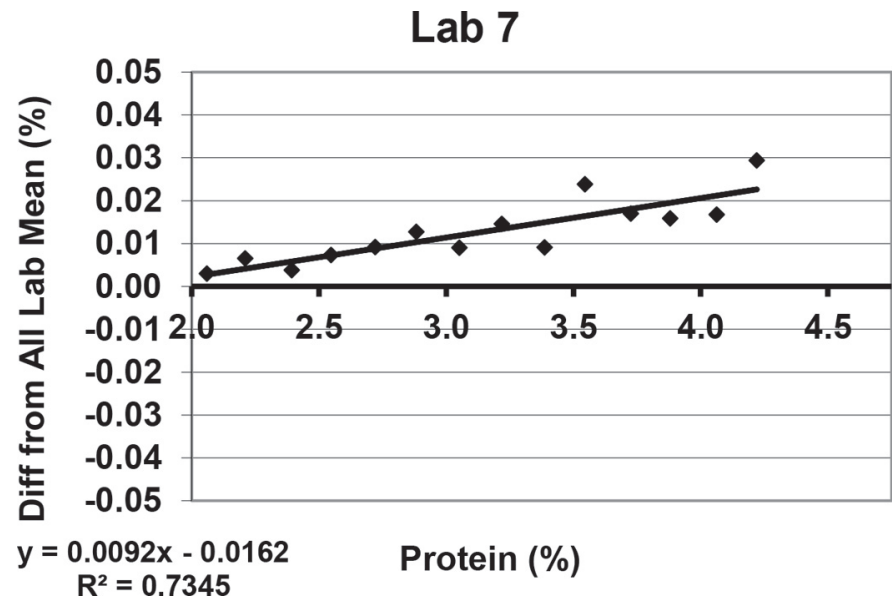

Figure 15. Residual plot of differences from the all-laboratory mean Kjeldahl true protein (AOAC method 991.22; AOAC International, 2000) result for laboratory 7 , as a function of true protein content of milk.

not match the value for the certificate and is different from the $0.1000 \mathrm{~N}$ nominal values for normality. This is a situation where in-house documentation of decision making and internal quality-control for traceability is very important in a certified laboratory.

The results of a sensitivity analysis to demonstrate the importance of this issue are given in Figure 16. This is an example where the laboratory uses 0.1000 $N$ for the titrant in the calculation of their results, but the true normality of the solution is not $0.1000 \mathrm{~N}$. The effect of the uncertainty in the titrant normality on the residual difference in protein value look exactly like what we see in the proficiency test data among different laboratories. It is interesting that this systematic error produces both a bias and slope of the residual plot of the protein data that is detected in our qualityassurance system for controlling the quality of reference protein testing for milk payment.

TS. Figures 17 and 18 are examples of graphs of the residual difference data (test value of the laboratory minus the all-laboratory mean with outliers excluded) for each sample for 1 analyst on 1 set of the 14 milks tested in duplicate (described above) plotted as a function of TS concentration in the milk. The forced-air, oven-drying method for measurement of the TS content of milk seems to be the most simple and straightforward method of the group of milk reference-testing methods. However, a variety of residual plot behaviors are observed, many of which have high coefficient of determination values. Residual plots with significant slopes (both positive, Figure 17, and negative, Figure 18) as a function of increasing solids concentration are observed and often have high coefficient of determination values. At first glance, this would appear to be an oven temperature issue or an issue of removal of residual moisture from the atmosphere within the drying oven. However, in extensive troubleshooting studies, we have not found evidence that the performance of the ovens is causing this behavior. Recently, we focused on weighing steps in the method and the condition and

\section{Influence of Uncertainty in Titrant Normality on KjeldahI Results}

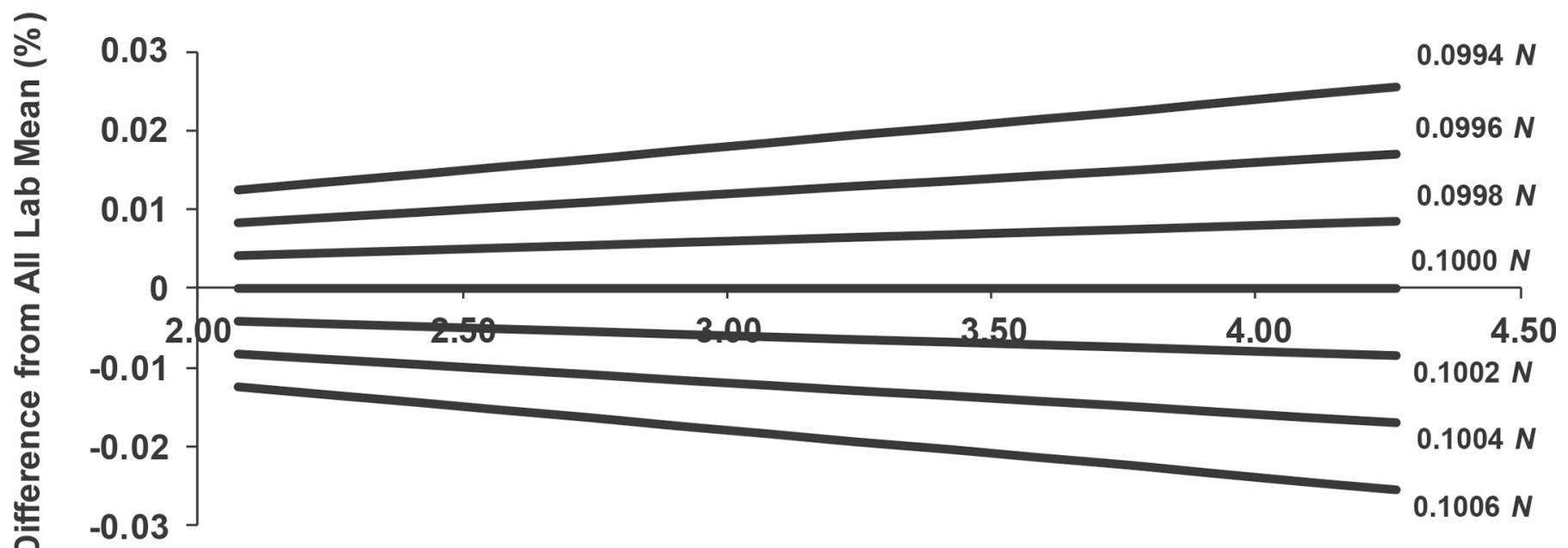

\section{True Protein (\%)}

Figure 16. Sensitivity analysis demonstrating the influence $0.1000 \mathrm{~N} \mathrm{HCl}$ titrant concentration uncertainty on the residual difference from the all-laboratory mean in a laboratory proficiency test evaluation using 14 milks over a range of protein from 2 to $4.3 \%$ true protein for the Kjeldahl method (AOAC method 991.22; AOAC International, 2000). 


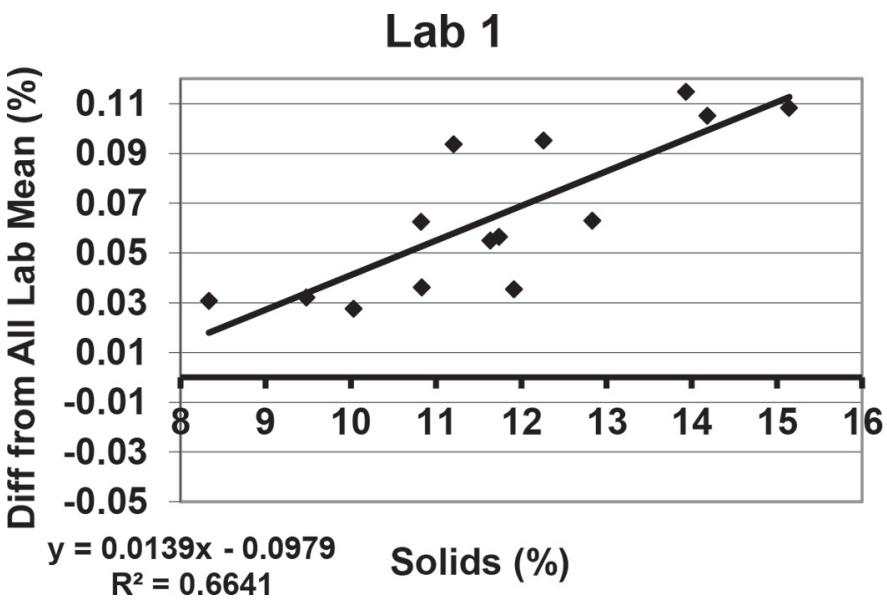

Figure 17. Residual plot of differences from the all-laboratory mean forced-air, oven-drying TS (AOAC method 990.20; AOAC International, 2000) result for laboratory 1 , as a function of TS content of milk.

method use of the analytical balance. A more in-depth discussion and presentation of data on the sources of error in the forced-air, oven-drying method will be the focus of a future publication.

\section{Proficiency Test Data and Samples for Calibration of a Secondary Test}

There is no question that the proficiency system described and the work invested in maintaining the performance of chemical reference-testing methods is large, but the economic impact of uncertainty in milk payment testing (particularly for fat and protein) is also very large (Lynch et al., 2004). Given the large investment in proficiency testing, it presented the opportunity to take

Lab 10

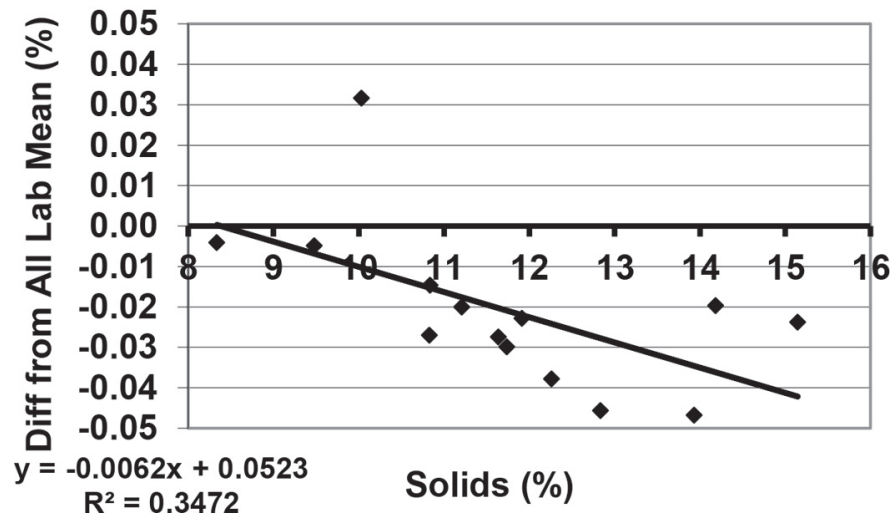

Figure 18. Residual plot of differences from the all-laboratory mean forced-air, oven-drying TS (AOAC method 990.20; AOAC International, 2000) result for laboratory 10, as a function of TS content of milk. advantage of using the work and financial investment to deliver more benefit to the dairy industry. Therefore, many additional sets of the proficiency test milks are made and the all-laboratory mean reference values produced in the proficiency testing are used as high-quality reference values for additional sets of the same milks. These milks are used as the basis of midinfrared milk analyzer calibration, as described by Kaylegian et al. (2006 a,b) and the International Diary Federation (2000).

\section{CONCLUSIONS}

We developed and demonstrated a multilaboratory combined proficiency testing and analytical method quality-assurance system as an approach to evaluate and improve the analytical performance of methods. To achieve this, a set of modified milks was developed and optimized to serve multiple purposes (i.e., proficiency testing, quality-assurance and method improvement, and to provide reference materials for calibration of secondary testing methods). Over a period of years, the approach has enabled the group of laboratories to document improved analytical performance (i.e., reduced within- and between-laboratory variation) of chemical reference methods used as the primary reference for calibration of high-speed electronic milk-testing equipment. An annual meeting of the laboratory technicians allows for review of results and discussion of each method and provides a forum for communication of experience and techniques that are of value to new analysts in the group. The monthly proficiency-testing sample exchanges have the added benefit of producing all-laboratory mean reference values for a set of 14 milks that can be used for calibration, evaluation, and troubleshooting of calibration adjustment issues on infrared milk analyzers.

\section{ACKNOWLEDGMENTS}

The authors thank the Test Procedures Committee of the USDA Federal Markets (Carrollton, TX) for financial support of this research, and the staff of the USDA Federal Milk Market Laboratories and laboratories under contract to the USDA Federal Milk Marketing Orders for their technical assistance in sample testing and communication of data.

\section{REFERENCES}

AOAC International. 2000. Official Methods of Analysis. 17th ed. AOAC International, Gaithersburg, MD.

Barbano, D. M., J. L. Clark, and C. E. Dunham. 1988. Comparison of the Babcock and ether extraction methods for determination of fat content of milk: Collaborative study. J. Assoc. Off. Anal. Chem. 71:898-914. 
Barbano, D. M., J. L. Clark, C. E. Dunham, and J. R. Fleming. 1990. Kjeldahl method for determination of total nitrogen content of milk: Collaborative study. J. AOAC Int. 73:849-859.

Barbano, D. M., J. M. Lynch, and J. Richard Fleming. 1991. Direct and indirect determination of true protein content of milk by Kjeldahl analysis: Collaborative study. J. Assoc. Off. Anal. Chem. $74: 281-288$.

Barbano, D. M., K. L. Wojciechowski, and J. M. Lynch. 2010. Impact of preservatives on the accuracy of milk component measurement by mid-infrared milk analyzers. J. Dairy Sci. 93:6000-6011.

Clark, J. L., D. M. Barbano, and C. E. Dunham. 1989. Comparison of two methods for determining total solids content of raw milk: Collaborative study. J. Assoc. Off. Anal. Chem. 72:712-718.

Geer, S. R., and D. M. Barbano. 2014a. Effect of colostrum on gravity separation of milk somatic cells in skim milk. J. Dairy Sci. 97:687-693.

Geer, S. R., and D. M. Barbano. 2014b. The effect of Ig and somatic cells on the gravity separation of fat, bacteria, and spores in pasteurized whole milk. J. Dairy Sci. 97:2027-2038.

Horwitz, W. 1995. Protocol for the design, conduct and interpretation of method-performance studies. Pure Appl. Chem. 67:331-343.

Hubbard, M. 1990. Statistical Quality Control for the Food Industry. Van Nostrand Reinhold, New York, NY.

International Dairy Federation. 2000. Whole milk: determination of milkfat, protein and lactose content - guidance on the operation of mid-infrared instruments. IDF Standard 141C:2000. Int. Dairy Fed., Brussels, Belgium.

Kaylegian, K. E, G. E. Houghton, J. M. Lynch, J. R. Fleming, and D. M. Barbano. 2006a. Calibration of infrared milk analyzers: Modified milk versus producer milk. J. Dairy Sci. 89:2817-2832.

Kaylegian, K. E., J. M. Lynch, G. E. Houghton, J. R. Fleming, and D. M. Barbano. 2006b. Modified versus producer milk calibration: Mid-infrared analyzer performance validation. J. Dairy Sci. $89: 2833-2845$
Lynch, J. M. 1998. Use of AOAC International performance statistics in the laboratory. J. AOAC Int. 81:679-684.

Lynch, J. M., and D. M. Barbano. 1999. Kjeldahl nitrogen analysis as a reference method for protein determination in dairy products. J. AOAC Int. 82:1389-1398.

Lynch, J. M., D. M. Barbano, and J. R. Fleming. 1997. Performance evaluation of direct forced-air total solids and Kjeldahl total nitrogen methods: 1990 through 1995. J. AOAC Int. 80:1038-1043.

Lynch, J. M., D. M. Barbano, and J. R. Fleming. 2007. Determination of lactose content of fluid milk by spectrophotometric enzymatic analysis using weight additions and path length adjustment: Collaborative study. J. AOAC Int. 90:196-216.

Lynch, J. M., D. M. Barbano, J. R. Fleming, and D Nicholson. 2004 Component testing, the dairy industry, and AOAC International. Pages 24-28 in Inside Laboratory Management, vol. 8. AOAC International, Gaithersburg, MD.

Lynch, J. M., D. M. Barbano, P. A. Healy, and J. R. Fleming. 1994. Performance evaluation of the Babcock and ether extraction methods: 1989 through 1992. J. AOAC Int. 77:976-981.

Lynch, J. M., D. M. Barbano, P. A. Healy, and J. R. Fleming. 2003. Effectiveness of temperature modification in decreasing the bias in milk fat test results between the Babcock and ether extraction methods. J. AOAC Int. 86:768-774.

Massart, D. L., B. G. M. Vandeginste, S. M. Deming, Y. Michotee, and L. Kaufman. 1988. Clustering techniques. Pages 371-375 in Chemometrics: A Textbook, Data Handling in Science and Technology. Vol. 2. Elsevier, New York, NY.

Thompson, M., and R. Wood. 1993. International harmonized proficiency testing of (Chemical) analytical laboratories. J. AOAC Int. 76:926-940.

USDA. 2000. Laboratory technical supplement, Federal Milk Market Administrator, Seattle, WA. Accessed May 6, 2016. http://www. fmmaseattle.com/lab/changes.htm 